\title{
Cohesin removal precedes Topoisomerase II decatenation at centromeres in male mammalian meiosis II
}

\author{
Rocío Gómez ${ }^{1}$, Alberto Viera ${ }^{1}$, Inés Berenguer ${ }^{1}$, Elena Llano ${ }^{2}$, Alberto M. Pendás ${ }^{2}$, José Luis \\ Barbero $^{3}$, Akihiko Kikuchi ${ }^{4}$ and José A. Suja ${ }^{1} *$
}

'Unidad de Biología Celular, Departamento de Biología, Facultad de Ciencias, Universidad Autónoma de Madrid, E-28049 Madrid, Spain

${ }^{2}$ Instituto de Biología Molecular y Celular del Cáncer (CSIC-USAL), Campus Miguel de Unamuno, E37007 Salamanca, Spain

${ }^{3}$ Departamento de Biología Celular y del Desarrollo, Centro de Investigaciones Biológicas, CSIC, E28040 Madrid, Spain

${ }^{4}$ Research Institute for Disease Mechanism and Control, Nagoya University Graduate School and Faculty of Medicine, Nagoya 466-8550, Japan

*Author for correspondence (jose.suja@uam.es)

Phone: 34914978240

Fax: 34914978344 


\section{Abstract}

Sister chromatid cohesion is regulated by cohesin complexes and Topoisomerase II $\alpha$. Although relevant studies have shed some light on the relationship between these two mechanisms of cohesion during mammalian mitosis, their interplay during mammalian meiosis remains unknown. In the present study, we have studied the dynamics of Topoisomerase II $\alpha$ in relation to that of the cohesin subunits RAD21 and REC8, the shugoshin SGOL2, and the helicase PICH, during both male mouse meiotic divisions. Our results strikingly show that Topoisomerase II $\alpha$ appears at stretched strands connecting the sister kinetochores of segregating early anaphase II chromatids, once the cohesin complexes have been removed from the centromeres. Moreover, the number and length of these Topoisomerase II $\alpha$ connecting strands increase between lagging chromatids at anaphase II after the chemical inhibition of the enzymatic activity of Topoisomerase II $\alpha$ by Etoposide. Our results also show that the ETinduced inhibition of Topoisomerase II $\alpha$ is not able to rescue the loss of centromere cohesion promoted by the absence of the shugoshin SGOL2 during anaphase I. Taking into account our results, we propose a two-step model for the sequential release of centromeric cohesion during male mammalian meiosis II. We suggest that the cohesin removal is a prerequisite for the posterior Topoisomerase II $\alpha$-mediated resolution of persisting catenations between segregating chromatids during anaphase II.

Keywords: Mouse, meiosis, DNA Topoisomerase II $\alpha$, cohesins, centromere, chromosome segregation 


\section{Introduction}

Faithful chromosome segregation during mitosis and meiosis requires that sister chromatids are intimately cohered from their replication at the interphase $\mathrm{S}$ phase until anaphase(s). During recent years it has become evident that sister chromatid cohesion (SCC) is maintained by cohesin protein complexes, and also by DNA catenations. The ring-shaped vertebrate mitotic cohesin complex consists of four subunits: two members of the Structural Maintenance of Chromosomes (SMCs) protein family (SMC1 $\alpha$ and SMC3), that heterodimerize, one kleisin subunit that closes the ring (RAD21), and a HEAT repeat domain protein (SA1/SA2) (Nasmyth 2011). During vertebrate mitosis the cohesin complexes are released from chromosomes in two steps. Throughout prophase most of the cohesin complexes at chromosome arms are released by phosphorylation of the subunit SA2 by the kinase PLK1, and the participation of some cohesin regulatory proteins (Hauf et al. 2005). Cohesin complexes at the centromere are protected from this dissociation by the complex shugoshin SGOL1/phosphatase PP2A (Kitajima et al. 2006, Riedel et al. 2006; Tang et al. 2006). Once all chromosomes are accurately bioriented, and the spindle-assembly checkpoint is silenced, the few cohesin complexes remaining at chromosome arms and those present at centromeres are released by proteolytic cleavage of RAD21 by separase during the metaphase/anaphase transition (Uhlmann et al. 1999). During meiosis, a specialized type of cell division characterized by two rounds of chromosome segregation after a single round of DNA replication to yield haploid gametes from diploid germ cells, SCC is also mediated by cohesin complexes. In mammalian meiosis, in addition to the canonical mitotic subunits SMC1 $\alpha$, SMC3, the kleisin RAD21, and SA1/SA2, several meiosis-specific cohesin subunits have been described. Thus, the meiotic paralogues of SMC1 $\alpha$ and SA1/SA2 are SMC1 $\beta$ and STAG3, respectively, while the kleisin RAD21 has two meiosis-specific paralogues, REC 8 and RAD21L (Gutiérrez-Caballero et al. 2011; Herrán et al. 2011; Ishiguro et al. 2011; Lee and Hirano 2011). Similar to vertebrate mitosis, SCC is released in two steps during meiosis (Suja and Barbero 2009; McNicoll et al. 2013). During mammalian meiosis I, cohesin complexes at chromosome arms are cleaved by separase during the metaphase I/anaphase I transition to allow the segregation of recombined homologous chromosomes. In this stage, the centromeric cohesin complexes are protected against separase cleavage by the complex shugoshin SGOL2/PP2A. During meiosis II, the SGOL2/PP2A complex that was protecting centromeric cohesin complexes redistributes, depending on tension, during metaphase II, and then the cohesin complexes are cleaved by separase to permit chromatid segregation (Gómez et al. 2007; Lee et al. 2008; Llano et al. 2008; GutiérrezCaballero et al. 2012).

The involvement of DNA catenations in holding sister chromatids together until the onset of anaphase was proposed some time ago (Murray and Szostak 1985). During DNA replication sister chromatids become catenated at multiple sites. These DNA catenations are resolved by DNA topoisomerase II $\alpha$ (Topo II $\alpha$ ), a homodimeric enzyme that introduces a transient double-strand break in one DNA molecule, then passes the sister DNA molecule through the transient break, and then reseals the break (Nitiss 2009a). Topo II $\alpha$ has a structural and/or enzymatic role in chromosome condensation since it is found at chromatid axes interacting with condensin complexes (Hirano 2012), and an essential enzymatic role in DNA replication, transcription and chromosome segregation (Porter and Farr 2004; Nitiss 2009a). It is known that Topo II $\alpha$ resolves most DNA catenations along chromosome arms in interphase and early stages of mitosis to allow sister chromatid resolution (Hirano 2012). However, DNA catenations persisting at the centromeres of metaphase chromosomes must be resolved during the metaphase/anaphase transition to allow sister chromatid segregation (Porter and Farr 2004; 
Lee and Bachant 2009). Indeed, early genetic and inhibition studies in different organisms showed that, in the (Porter and Farr 2004; Lee and Bachant 2009). More recent studies using RNAi knockdown of Topo II $\alpha$ support those early observations, and in fact Topo II $\alpha$ cleavage activity at active centromeres has been demonstrated in human chromosomes (Lee and Bachant 2009). The relevance of Topo II $\alpha$ in SCC has been reinforced by the identification of the helicases PICH (Baumann et al. 2007; Spence et al. 2007; Wang et al. 2008; Ke et al. 2011; Kaulich et al. 2012) and BLM (Chan et al. 2007; Ke et al. 2011) at centromeric ultrafine anaphase bridges thought to contain unresolved DNA catenations in all cultured normal cells tested. Interestingly, it has also been proposed that centromere DNA decatenation by Topo II $\alpha$ during early anaphase depends on previous cohesin removal (Wang et al. 2010). In this regard, inhibition or depletion of Topo II $\alpha$ rescues the cohesion defects of cohesin-depleted vertebrate cells (Vagnarelli et al. 2004; Toyoda and Yanagida 2006).

The exact role of Topo II $\alpha$ during mammalian meiotic divisions is largely unknown. Its role during mammalian meiosis has been analysed by inhibiting its function mainly with Etoposide (ET). This is a potent genotoxic Topo II $\alpha$ inhibitor used in cancer therapy, whose effect lies in blocking the activity of Topo II $\alpha$ once it has cut the concatenated DNA molecules, but prior to the occurrence of the religation (Nitiss 2009b). It has been reported that ET-mediated inhibition of Topo II $\alpha$ during mouse meiosis induces alterations on homologous recombination during prophase I, fragmentation of centromeric satellite DNA, and failures in chromosome segregation during meiotic divisions (Kallio and Lähdetie 1996; Marchetti et al. 2001; Russell et al. 2004, Matulis and Handel 2006). Altogether, these data suggest that Topo II $\alpha$ has a role in chromosome segregation during mouse meiotic divisions, as in mitosis. However, the spatial and temporal distribution of Topo II $\alpha$ during both meiotic divisions is unknown. Moreover, the exact nature of the ET-induced alterations in chromosome segregation during both mammalian meiotic divisions has not been analysed. Thus, although the regulation of cohesin removal during mammalian meiosis is relatively well-studied, the decatenating role of Topo II $\alpha$ is at present unclear. Thus, we need additional information on how cohesin-dependent and Topo II $\alpha$-dependent SCC are coordinated during both mammalian meiotic divisions. On the other hand, controversial results about Topo II distribution have been previously reported in male mouse (Cobb et al. 1999), cock (Moens and Earnshaw 1989) and Bombyx mori (Moens 1990) spermatocytes. For this, and in order to address these discrepancies, a complete analysis of Topo II $\alpha$ distribution in male mouse meiosis is needed.

In the present study, we first examined by immunofluorescence the subcellular distribution of Topo II $\alpha$ during each stage of male mouse meiosis. We also analysed the distribution of Topo II $\alpha$ with the cohesin subunits RAD21 and REC8 in order to establish their relative dynamics during both meiotic divisions. Since Topo II $\alpha$ was particularly enriched at the centromere, we also studied its pattern of distribution by comparing with that of kinetochores, three important inner centromere proteins, the shugoshin SGOL2, the microtubule depolymerase MCAK, and the chromosomal passenger complex protein INCENP, and also the helicase PICH. To obtain needed additional information concerning the role of Topo II $\alpha$ during male mammalian meiosis we inhibited its function with ET and analysed the induced alterations in chromosome segregation. Finally, we tested the behaviour of Topo II $\alpha$ in a genetic mouse model of premature loss of cohesion in meiosis I (Sgol2 ${ }^{-/}$ knockout) (Llano et al. 2008), and also whether ET could rescue the defective centromere cohesion in this system. Our results lead us to propose a model that explains the temporal and spatial relationship between cohesin complexes and Topo II $\alpha$ at the centromeres during the second meiotic division. We propose that there is 
a sequential removal of cohesin complexes followed by Topo II $\alpha$-mediated decatenation resolution during early anaphase II. 


\section{Materials and methods}

\section{Squashing of seminiferous tubules and spreading of spermatocytes}

Testes from adult male C57BL/6, Sgol2 ${ }^{-/}$(Llano et al. 2008) and REC8-myc transgenic mice (Kudo et al. 2006) were used for this study. All animals were handled in strict accordance with good animal practice as defined by the relevant national and/or local animal welfare bodies, and all animal work was approved by the UAM committee. Testes were removed, detunicated, and seminiferous tubules then processed for either squashing or spreading techniques. For squashing, we followed the technique previously described (Page et al. 1998; Parra et al. 2002). Briefly, seminiferous tubules were fixed in freshly prepared $2 \%$ formaldehyde in PBS (137 mM NaCl, $2.7 \mathrm{mM} \mathrm{KCl}, 10.1 \mathrm{mM} \mathrm{Na}_{2} \mathrm{HPO}_{4}, 1.7 \mathrm{mM} \mathrm{KH} \mathrm{PO}_{4}, \mathrm{pH}$ 7.4) containing 0.05\% Triton X-100 (Sigma). After 5 minutes, several seminiferous tubules fragments were placed on a slide coated with $1 \mathrm{mg} / \mathrm{ml}$ poly-L-lysine (Sigma) with a small drop of fixative, and gently minced with tweezers. The tubules were then squashed and the coverslip removed after freezing in liquid nitrogen. For the immunolabelling techniques we proceeded as previously described (Parra et al. 2004). For spreading of spermatocytes, we followed the drying-down technique previously described (Peters et al. 1997).

\section{Cell line and culture}

Pam212 mouse cells were grown on $22 \mathrm{~mm}$ square glass coverslips placed into dishes, using Dulbecco's modified Eagle's medium (DMEM) containing 10\% (v/v) fetal calf serum, $50 \mathrm{U} / \mathrm{ml}$ penicillin, $50 \mathrm{mg} / \mathrm{ml}$ streptomycin, and 1\% (v/v) $0.2 \mathrm{M} \mathrm{L-glutamine} \mathrm{(all} \mathrm{from} \mathrm{Gibco).} \mathrm{Cells} \mathrm{were} \mathrm{fixed} \mathrm{in} \mathrm{2 \%} \mathrm{formaldehyde} \mathrm{in} \mathrm{PBS}$ for 10 minutes, and then processed for immunofluorescence.

\section{Immunofluorescence microscopy}

After fixation, the squashed or spreaded spermatocyte preparations were rinsed three times for 5 minutes in PBS, and incubated overnight at $4^{\circ} \mathrm{C}$ with the corresponding primary antibodies diluted in PBS. In double-labelling experiments, primary antibodies from different host species were incubated simultaneously. Following three washes in PBS for 5 minutes, the slides were incubated for 30 minutes at room temperature with the corresponding secondary antibodies. The slides were subsequently rinsed in PBS and counterstained for 3 minutes with $10 \mu \mathrm{g} / \mathrm{ml}$ DAPI (4',6-diamidino-2-phenylindole). After a final rinse in PBS, the slides were mounted with Vectashield (Vector Laboratories) and sealed with nail polish.

Immunofluorescence image stacks were collected on an Olympus BX61 microscope equipped with epifluorescence optics, a motorized z-drive, and an Olympus DP70 digital camera controlled by analySIS software (Soft Imaging System). Stacks were analysed and processed using the public domain ImageJ software (National Institutes of Health, USA; http://rsb.info.nih.gov/ij). Final images were processed with Adobe Photoshop 7.0 software.

\section{Antibodies}

For immunofluorescence staining, the following primary antibodies were used at the indicated dilution: mouse monoclonal 4E12 antibody against human Topo II $\alpha$ (Cobb et al. 1999) at 1:10; rabbit polyclonal antibody against human Topo II $\alpha$ (TopoGEN; cat. 2011-1) at 1:10; rabbit polyclonal antibody against mouse SYCP3 (Abcam, ab-15092) at 1:100; sheep polyclonal antibody against human MCAK (a gift of Linda Wordeman; 
Andrews et al. 2004) at 1:40; rabbit polyclonal antibody (pAb1186) raised against chicken INCENP which also recognizes mouse INCENP (Parra et al. 2003) (a gift of Bill Earnshaw; Eckley et al. 1997) at 1:100; rabbit polyclonal antibody against the C-terminus of mouse REC8 (a gift of Jibak Lee; Lee et al. 2003) at 1:10; rabbit serum K854 against human RAD21 (Prieto et al. 2002) at 1:50; rabbit serum K1059 against mouse SGOL2 (Gómez et al. 2007) at 1:20; human anti-centromere autoantibody (ACA serum) revealing kinetochores (Antibodies Incorporated, 15-235) at 1:50; and rabbit antibody against human PICH (A0201) (a gift of Erich Nigg; Wang et al. 2008) at 1:5. REC8-myc was revealed with a rabbit polyclonal antibody against the myc epitope tag (Upstate, 06-549) at a 1:10 dilution.

The secondary antibodies used were: donkey anti-human IgG (Jackson), donkey anti-rabbit IgG (Jackson), donkey anti-sheep (Jackson), and donkey anti-mouse IgG (Jackson). All of them were employed at a 1:150 dilution in PBS, and were conjugated with either Texas Red or fluorescein isothiocyanate (FITC).

\section{Inhibition of Topo II $\alpha$ activity by Etoposide}

We prepared $50 \mathrm{mg} / \mathrm{kg}$ solutions of Etoposide (ET) (Sigma-Aldrich, E1383) that correspond to 12,5 $\mathrm{mg} / \mathrm{ml}$ diluted in dimethyl sulfoxide (DMSO) (Sigma-Aldrich, D5879). After tempering the drug to body temperature, we then administrated the corresponding volume of drug by intraperitoneal injections according to the mice weight. Fifteen wild-type C57BL/6 adults aged 2-3 months were used. Specimens were monitored and euthanized by cervical dislocation after 5,24 or 48 hours following all the recommendations of the Animal Experimentation UAM Ethics Committee. After conducting various tests to accurate optimal ET concentration (concentration tests for $60 \mathrm{mg} / \mathrm{kg}, 80 \mathrm{mg} / \mathrm{kg}, 100 \mathrm{mg} / \mathrm{kg}$ and $200 \mathrm{mg} / \mathrm{kg}$ ET in DMSO), we concluded that the optimal ET concentration for this study was $50 \mathrm{mg} / \mathrm{kg}$, verifying that progressively higher concentrations caused the same effects, but higher rates of cell death. No mice died after the treatment. Control tests were also performed in individuals exclusively injected with the ET solvent DMSO, to check for putative effects on meiotic divisions. Our results demonstrated that DMSO did not affect meiotic divisions. Similarly, $50 \mathrm{mg} / \mathrm{kg}$ ET was administered intraperitoneally for 24 hours to $\mathrm{Sgol}^{-/}$mice.

\section{TUNEL assay}

The DNA fragmentation-associated apoptosis of ET-treated spermatocytes was detected by the TdT-mediated dUTPfluorescein nick end labelling (TUNEL) assay by using a kit (Roche, 11684795910) according to manufacturer's protocol. Nuclei were counterstained for 3 minutes with $10 \mu \mathrm{g} / \mathrm{ml}$ DAPI. Tests were developed on formaldehydefixed squashed spermatocytes of control samples and 5 hours, 24 hours and 48 hours after ET exposure. 


\section{Results}

\section{Topo II $\alpha$ appears at the centromeres and chromatid axes during the first meiotic division}

To analyse the pattern of distribution of Topo II $\alpha$ during both meiotic divisions we double-immunolocalised it with SYCP3 and with the kinetochores (ACA serum) on squashed spermatocytes (Fig. 1). We used the squashing technique since it does not disturb chromosome condensation and distribution in dividing spermatocytes, and also allows the differentiation of all meiotic stages (Parra et al. 2002; Gómez et al. 2007). The labelling of SYCP3, a structural protein of the Axial Elements/Lateral Elements (AEs/LEs) of the Synaptonemal Complex (SC), was used to define the meiotic staging during the first meiotic division.

During prophase I, Topo II $\alpha$ was detected at the chromocentres from leptonema onwards as previously reported (Cobb et al. 1999) (data not shown). The chromocentres, that represent clustered centromere heterochromatic regions from different chromosomes, and that are clearly discerned after DAPI staining, are located at the nuclear periphery. However, since we projected different focal planes throughout the spermatocytes, some chromocentres appeared at the nuclear interior. In this regard, Topo II $\alpha$ was located at the centromeric end of pachynema SCs as detected with the anti-SYCP3 antibody (Fig. 1a) as previously reported (Cobb et al. 1999). Each Topo II $\alpha$-labelled chromocentre showed several kinetochore signals inside them (Fig. 1b-d). Staining of Topo II $\alpha$ along AE/LEs of the SC was never detected. In metaphase I, when all the bivalents are bioriented, side-views of the bivalents showed that SYCP3 and Topo II $\alpha$ partially colocalised at the centromeres, but while SYCP3 arranged as a T-shaped signal (Parra et al. 2004; Parra et al. 2006), two rounded signals of Topo II $\alpha$ were observed (Fig. 1e, Fig. S1a, b). In a more detailed analysis of top-viewed centromeres we detected that SYCP3 appeared as two closely associated rings that surrounded the two masses of Topo II $\alpha$ (inserts in Fig. 1e). In addition to the strong labelling at centromeres, Topo II $\alpha$ was also detected as faint longitudinal signals along the condensed chromatids representing the chromatid axes (Viera et al. 2007). In selected metaphase I bivalents, we observed that SYCP3, by contrast, was present as a series of faint and small patches at the region of contact between the sister chromatids, the so-called interchromatid domain (Prieto et al. 2001; Parra et al. 2004) (insert in Fig. 1e, Fig. S1a,b). By analysing the relative distribution of Topo II $\alpha$ and the kinetochores we found that the Topo II $\alpha$ centromeric signals appeared as two rounded masses below the closely associated sister kinetochores (Fig. 1f-h and Supplementary Movie 1). In anaphase I chromosomes, Topo II $\alpha$ was still present at the centromeres and chromatid axes while SYCP3 was only detected at the centromeres (Fig. 1i-1, Fig. S1c). During telophase I, neither SYCP3 nor Topo II $\alpha$ were detected at chromosome arms. However, Topo II $\alpha$ signals at centromeres persisted partially colocalising with SYCP3 bars (Parra et al. 2004) (Fig. 1m), and below the kinetochore signals (Fig. 1n-p).

\section{Topo II $\alpha$ and cohesin occupy different subdomains at metaphase I centromeres}

In order to gain insight into the centromere distribution of Topo II $\alpha$ during metaphase I, we made doubleimmunolabellings of Topo II $\alpha$ with either the shugoshin SGOL2 or the microtubule depolymerase MCAK (Mitotic Centromere-Associated Kinesin) since it has been previously reported that they are markers of the inner centromere domain (Parra et al. 2006; Gómez et al. 2007; Llano et al. 2008). Our results showed that the two round and closely associated centromere signals of Topo II $\alpha$ partially colocalised with SGOL2 and MCAK (Fig. 2a-d), that showed a similar T-shaped distribution at the inner centromere domain. A similar result was observed when the relative distributions of Topo II $\alpha$ and the cohesin subunit RAD21 (Parra et al. 2004; Gómez et al. 
2007) was compared at metaphase I centromeres (Fig. 2 e, f). By contrast, the meiosis-specific cohesin subunit REC8 showed a comma-like distribution at centromeres as previously reported (Gómez et al. 2007) in between the two large Topo II $\alpha$ signals (Fig. $2 \mathrm{~g}$, h). Additionally, Topo II $\alpha$ was detected at the chromatid axes flanking the labelling of RAD21 (Fig. 2e, f), REC8 (Fig. 2g, h), and SYCP3 (Fig. S1a, b) at the interchromatid domain. These results indicate that Topo II $\alpha$ and the cohesin subunits RAD21 and REC 8 occupy different subdomains at the inner domain of metaphase I centromeres.

\section{Topo II localises at thin strands connecting the centromeres of early anaphase II segregating chromatids}

During interkinesis, an interphase stage without DNA replication between the two meiotic divisions, Topo II $\alpha$ was present at the large internal chromocentres that were also clearly observed after DAPI staining, and where the kinetochores were found (Fig. 3a-c). In prophase II, Topo II $\alpha$ was also detected at chromocentres representing clustered centromeres of condensing chromosomes (Fig. 3d-f). During metaphase II, when all the chromosomes were aligned at the metaphase plate, Topo II $\alpha$ was detected at the centromeres. In this sense, Topo II $\alpha$ appeared as one large signal below each sister kinetochore, and as a strand connecting them (Fig. 3g-I and Supplementary Movie 2). As a rule, these Topo II $\alpha$ connecting strands (TOCOSs) at the inner centromeric domain were thinner than the signals below each kinetochore (inserts in Fig. 3h). Surprisingly, during early anaphase II, once chromatids had begun their migration to opposite poles, the TOCOSs persisted and appeared stretched between the separating sister kinetochores (Fig. 3j-1 and Supplementary Movie 3). These TOCOSs were no longer observed by late anaphase II and telophase II although Topo II $\alpha$ signals were still detected below each kinetochore (Fig. 3m-o). These observations indicate that Topo II $\alpha$ provides a persisting link between the two separating centromeres during early anaphase II.

\section{TOCOSs persist between the centromeres of early anaphase II chromatids after the release of cohesin complexes}

Since TOCOSs persisted in early anaphase II, and this is an unprecedented and outstanding observation, we compared their behaviour in relation to SGOL2, MCAK, or the chromosomal passenger complex protein INCENP. We selected these proteins for comparative purposes because, it has been reported that during chromosome congression to the metaphase II plate, these proteins redistribute from a band connecting sister kinetochores to the kinetochore region, once chromosomes have attained a bipolar orientation and their centromeres are under tension to opposite poles (Parra et al. 2003; Parra et al. 2006; Gómez et al. 2007). Our results showed that at metaphase II, when all chromosomes are aligned and under tension, SGOL2 and MCAK signals appeared as pairs of dots at kinetochore regions (Parra et al. 2006; Gómez et al. 2007) while bright Topo II $\alpha$ signals were apparent below them and also at TOCOSs (Fig. 4a-d). Likewise, TOCOSs persisted during chromosome congression to the metaphase II plate while INCENP redistributed from a band traversing the inner centromere domain (Fig. S2a, b) to single signals at the kinetochore regions (Fig. S2c, d) (Parra et al. 2003).

To further define the temporal behaviour of TOCOSs in relation to cohesins, we then analysed the relative distribution of Topo II $\alpha$ and the cohesin subunits RAD21 or REC8 in metaphase II and anaphase II spermatocytes (Fig. 4e-j). As previously reported, we were not able to detect RAD21 labelling at metaphase II centromeres (Parra et al. 2004) (Fig. 4e, f). The antibody that we used to reveal REC8 did not render any kind of signal at metaphase II centromeres in squashed spermatocytes. However, when we detected REC 8 on metaphase II spermatocytes from mice expressing REC8-myc, a single REC8 signal was observed colocalising with the 
central region of TOCOSs (Fig. 4g, h). Strikingly, REC8 was not detected in anaphase II spermatocytes although stretched TOCOSs colocalised with thin chromatin threads between segregating chromatids (Fig. 4i, j). These results suggest that the release of the cohesin REC8 from metaphase II centromeres allows the initial segregation of chromatids to opposite poles, and that the putative decatenation activity of Topo II $\alpha$ occurs later during early anaphase II.

\section{Topo II $\alpha$ and PICH dynamics is different during meiosis II and mitosis}

Since it has been reported that the helicase PICH appears at centromeric ultrafine anaphase bridges in somatic cultured cells (Baumann et al. 2007; Spence et al. 2007; Wang et al. 2008; Ke et al. 2011; Kaulich et al. 2012), we studied the relative distribution of PICH and Topo II $\alpha$ during the metaphase II/anaphase II transition to assess whether they colocalised at TOCOSs. By using an antibody against human PICH we were not able to detect centromere signals during the second meiotic division. However, in Pam212 mouse somatic cells the same antibody revealed long PICH strands between the centromeres of segregating chromatids during early anaphase (Fig. S3). Interestingly, we did not find TOCOSs in these same early anaphase cells. As during spermatogonial mitosis, Topo II $\alpha$ was only detected at the centromeres and at the chromatid axes (Fig. S4). Altogether, our data suggest that the decatenation activity of Topo II $\alpha$ is differentially regulated during mitotic anaphase and meiotic anaphase II.

\section{Inhibition of Topo II $\alpha$ activity promotes chromosome lagging during meiotic divisions}

We then analysed how the inhibition of Topo II $\alpha$ activity with $50 \mathrm{mg} / \mathrm{kg}$ of ET could affect meiotic divisions after 5, 24 and $48 \mathrm{~h}$ of treatment. ET is a Topo II $\alpha$ inhibitor, whose effect lies in blocking the activity of Topo II $\alpha$ once it has cut the concatenated DNA molecules, but prior to the occurrence of the religation. Since it is known that ET does not disturb the chromosomal Topo II $\alpha$ distribution, but only its enzymatic activity (Nitiss 2009b), we made double-immunolabellings of Topo II $\alpha$ and kinetochores on treated spermatocytes to visualize putative abnormalities during meiotic divisions. The intraperitoneal administration of DMSO demonstrated that the ET solvent did not affect the progression and presence of meiotic divisions since in all metaphase I and II spermatocytes all the bivalents/chromosomes were perfectly aligned; the percentage of univalents in metaphase I were the same as in untreated WT spermatocytes (around 0,5\%, most of the times the sex univalents); and we did not found anaphase bridges in anaphase I or II. Quantitative data of ET-treated spermatocytes are shown in Fig. 5. After ET treatment, no apparent aberrations could be observed during prophase I stages after following the pairing and synapsis processes with anti-SYCP3. However, in metaphase I spermatocytes, although chromosome condensation was not affected, we found different effects on chromosome behaviour and appearance that allowed us to classify them into several categories. In this sense, we found two main categories of metaphase I spermatocytes, alive with normally condensed bivalents (Fig. S5a), and death ones with hypercompacted chromosomes that did not show immunoreactivity (Fig. S5b-f). In order to know whether these last degenerating metaphase I spermatocytes were undergoing apoptosis, we carried out a TUNEL assay on squashed spermatocytes. We indeed found that all these death spermatocytes were apoptotic (Fig. S6), in line with previous findings showing that dividing spermatocytes showing hypercondensed chromosomes are apoptotic (Adelman and Petrini 2008). The percentage of alive metaphase I spermatocytes progressively decreased with increasing times of ET treatment and accordingly that of apoptotic metaphase I spermatocytes increased (Fig. 
5a). Among alive metaphase I spermatocytes we found three phenotypes: type I representing normal spermatocytes with aligned bivalents (Fig. S5a), type II that included metaphase I spermatocytes with univalents, as a rule the sex chromosomes (Fig. 6a-d), and type III that comprised metaphase I spermatocytes with misaligned bivalents. Regarding the type II phenotype it is worth to mention that we were able to recognise univalents since they were not aligned at the metaphase I plate, and they showed kinetochores only at one of their ends. Our results showed that a prolonged ET treatment promoted a decrease in the percentage of alive type I metaphase I spermatocytes, while the percentage of those of type II decreased and the percentage of those of type III slightly increased (Fig. 5a). Interestingly, the decrease in the percentage of alive type II spermatocytes correlated with the increase in the percentage of apoptotic metaphase I spermatocytes with univalents (Fig. 5a, Fig. S5c, d, f). Likewise, the overall decrease in the percentage of type I and III alive spermatocytes correlated with the increase in the percentage of apoptotic ones without univalents (Fig. 5a, Fig. S5b, e). These results indicate that ET promotes misalignment of bivalents and the appearance of univalents, and that metaphase I spermatocytes with such aberrant chromosome behaviours enter an apoptotic pathway.

In ET-treated individuals, some of the alive anaphase I/telophase I spermatocytes showed one to four lagging chromosomes with clear chromatin bridges (Fig. 5b). Interestingly, the anaphase I/telophase I bridges were labelled with the anti-Topo II $\alpha$ antibody (Fig. 6e-1 and Supplementary Movie 4). With prolonged times of ET treatment the percentage of alive anaphase I/telophase I spermatocytes with chromatin bridges decreased and this was concomitant with an increase in the percentage of apoptotic spermatocytes with and without bridges (Fig. 5b, Fig. S5g, h).

ET also showed an effect on chromosome alignment and segregation during the second meiotic division. Thus, although most alive metaphase II spermatocytes were normal (Fig. S5k), a few percentage showed misaligned chromosomes (Figs. 5c and 7a-d). On the other hand, with a prolonged ET exposure the percentage of apoptotic metaphase II spermatocytes increased dramatically (Fig. 5c, Fig. S51-o). During anaphase II/telophase II, as seen during the first division, the percentage of alive spermatocytes with lagging chromosomes and chromatin bridges was relatively high at 5 hours of ET treatment and then decreased with a concomitant increase in the percentage of apoptotic spermatocytes with and without bridges at 48 hours (Fig. 5d, Fig. S5p-y). One remarkable observation was that in alive anaphase II/telophase II spermatocytes the chromatin bridges that we detected were labelled with Topo II $\alpha$ and also included chromatin as revealed with DAPI (Fig. 7e-n and Supplementary Movies 5-7). A careful inspection showed that these Topo II $\alpha$ bridges connected the sister kinetochores of lagging chromatids (Fig. 7i-n). This situation is reminiscent of the TOCOSs that stretched during normal early anaphase II spermatocytes and that finally disappeared. Taken together, these results indicate that the chemical inhibition of Topo II $\alpha$ activity leads to the formation of anaphase bridges between lagging chromosomes.

\section{Topo II $\alpha$ does not maintain centromere cohesion during meiosis I in the absence of cohesin}

It has been previously described that in knockout mutants for the shugoshin SGOL2 cohesion disappears not only from chromosome arms, but also from centromeres, during the first meiotic division. As a consequence, in metaphase II-like spermatocytes all chromatids appear individualised (Llano et al. 2008). In order to know whether the single chromatids found in these metaphase II-like spermatocytes are still connected by TOCOSs in the absence of centromeric cohesins, we made a double immunolabelling of Topo II $\alpha$ and kinetochores on Sgol2- 
/. mutant spermatocytes. Our results showed that Topo II $\alpha$ appeared accumulated at the centromeres below the closely associated sister kinetochores in metaphase I bivalents as in normal metaphase I spermatocytes (compare Figs. 1f-h and $8 \mathrm{a}, \mathrm{b})$. In anaphase I mutant spermatocytes, and although sister kinetochores had prematurely lost their association and appeared separated, Topo II $\alpha$ was still evident below, but not between them (Fig. 8e, f). Likewise, a centromere Topo II $\alpha$ signal was evident below each kinetochore in the forty individual chromatids found in metaphase II-like spermatocytes, and no TOCOSs were detected between those chromatids (Fig. 8i, j). These results support that in the absence of SGOL2 the centromere cohesion mediated by cohesin complexes is lost by anaphase I/telophase I, and suggest that sister catenations mediated by Topo II $\alpha$ have also apparently disappeared.

Next, we tried to elucidate whether the inhibition of Topo II $\alpha$ by ET could rescue the loss of centromere cohesion in the absence of SGOL2 during anaphase I/telophase I. For this, we analysed chromosome behaviour in ET-treated Sgol2 ${ }^{-/}$mutant spermatocytes. Our results showed that in anaphase I mutant spermatocytes sister kinetochores were separated but not connected by TOCOSs (Fig. 8g, h). Likewise, in treated metaphase II-like spermatocytes all the chromatids were individualised and TOCOSs among them were never observed, as in untreated metaphase II-like spermatocytes (Fig. 8k, 1). Altogether, these results indicate that the inhibition of Topo II $\alpha$ is not able to rescue the loss of SCC in the absence of centromere cohesins during anaphase I/telophase I. 


\section{Discussion}

During vertebrate mitosis SCC is maintained by cohesin complexes and DNA catenations. Recent data suggest that centromeric cohesin complexes are cleaved and released during the metaphase/anaphase transition, and that DNA catenations at centromeres are then resolved by Topo II $\alpha$ during early anaphase (Wang et al. 2010). However, it remains unexplored whether this phenomenon is also conserved during mammalian meiosis II. To evaluate the conservation of this phenomenon during mammalian meiosis we have first analysed the relative pattern of distribution of Topo II $\alpha$ and cohesin subunits during both meiotic divisions in male mice. We have also chemically inhibited the enzymatic activity of Topo II $\alpha$ with ET to determine the nature of putative alterations in chromosome segregation. Moreover, we have also studied the behaviour of Topo II $\alpha$ in the absence of centromeric cohesin complexes during meiosis II in mice knockout for the shugoshin SGOL2 and also treated with ET.

\section{Topo II $\alpha$ does not have an essential role in maintaining centromere and arm cohesion during the first meiotic} division

Our analysis in male mouse meiosis on squashed and spread spermatocytes demonstrates that Topo II $\alpha$ is only detected at the chromocentres during prophase I, as previously reported (Cobb et al. 1999). Hence, our data are in contrast with other reports on cock (Moens and Earnshaw 1989), yeast (Klein et al. 1992) and Bombyx mori meiocytes (Moens 1990), since we have never detected Topo II $\alpha$ along the LEs of the SC. From prophase I onwards, there are no published data showing in detail the localization pattern of Topo II $\alpha$ in mammalian meiosis. Our results show that Topo II $\alpha$ is found at the centromeres of metaphase I chromosomes as two signals below sister kinetochores, and that these signals exceed laterally the signals of the inner centromere proteins shugoshin SGOL2 (Gómez et al. 2007), and MCAK (Parra et al. 2006). Moreover, Topo II $\alpha$ signals also exceed those of the cohesin RAD21, while the centromeric accumulation of REC8 lied in between the Topo II $\alpha$ signals. Thus, Topo II $\alpha$ and cohesin subunits occupy different subdomains at the inner centromere (Fig. 9). The finding that in ET-treated metaphase I and anaphase I spermatocytes the chromatids are still associated at their centromere regions, indicate that Topo II $\alpha$ has not an essential enzymatic role in maintaining centromere cohesion during the first meiotic division. Moreover, in the absence of SGOL2, centromere cohesion is lost during the anaphase I/telophase I transition (Llano et al. 2008), and this defect is not rescued by ET-induced inhibition of Topo II $\alpha$. This result also supports that Topo II $\alpha$ has no a role in the maintenance of centromere cohesion. Our results indicate that the cohesin complexes are the essential executors for maintaining centromere cohesion and that Topo II $\alpha$ might have a structural role at the heterochromatic centromere regions in maintaining their topology during meiosis I. Likewise, the presence of Topo II $\alpha$ along the chromatid axes of metaphase I bivalents, and not at the interchromatid domain where the cohesin subunits RAD21 and REC8 are located, indicate that Topo II $\alpha$ is not directly involved in maintaining arm cohesion during meiosis I. Our observation that Topo II $\alpha$ is still present at the chromatid axes in anaphase I chromosomes, when arm cohesion has disappeared, also supports this conclusion.

\section{Topo II $\alpha$ role in meiotic recombination and chromosome segregation during the first meiotic division}

Our results show that the inhibition of Topo II $\alpha$ by ET induces the appearance of univalents and misaligned bivalents in metaphase I spermatocytes, that mostly enter an apoptotic pathway with increasing times of ET 
treatment. The presence of univalents in these ET-treated metaphase I spermatocytes suggests that Topo II $\alpha$ may metaphase I univalents found after ET treatment are the X and Y sex chromosomes. Since sex chromosomes are under different regulation compared to autosomes in terms of condensation, gene silencing, histone modification and synapsis events, perhaps the regulation of recombination events within the sex pair are differentially regulated by instance by Topo II $\alpha$.

We additionally observed that in ET-treated individuals, anaphase I/telophase I spermatocytes showed a high incidence of lagging chromosomes, a predominant phenotype in anaphase when Topo II $\alpha$ function is deficient during mitosis (Porter and Farr 2004). Interestingly, we found that lagging chromosomes were connected by Topo II $\alpha$-labelled chromatin bridges that, as a rule, appeared close to the centromeres. Since we are proposing that Topo II $\alpha$ does not have a role in SCC, the presence of chromatin bridges between lagging chromosomes during anaphase I indicate that ET could induce DNA damage that would impair an accurate chromosome segregation during anaphase I (Kallio and Lähdetie 1996; Marchetti et al. 2001). The increase in the percentage of apoptotic anaphase I/telophase I spermatocytes with increasing times of ET treatment indicates that most of the spermatocytes with lagging chromosomes enter an apoptotic pathway. Altogether, our data suggest that Topo II $\alpha$ has a role in chromosome segregation during mouse meiosis I.

\section{Topo II $\alpha$ decatenation at centromeres after removal of cohesin complexes during meiosis II}

One of the most outstanding results obtained in the present study refers to the appearance of TOCOSs between the sister kinetochores at the inner domain of metaphase II centromeres. This previously unknown pattern of distribution of Topo II $\alpha$ at TOCOSs is reminiscent of the dense strands connecting sister kinetochores observed by electron microscopy with the Os-PPD cytochemical technique at mouse metaphase II centromeres (Parra et al. 2006). Moreover, similar connecting strands have been observed by electron microscopy at metaphase II centromeres in the field vole Microtus agrestis (Wolf et al. 1988). Thus, Topo II $\alpha$ seems to be one of the protein components at these structural connecting strands at the inner domain of male mammalian metaphase II centromeres. In light of sex-specific differences in mammalian meiotic progression and control, our conclusions should be clearly anchored in the context of spermatogenesis, and should be corroborated in females. We have found that TOCOSs persist at the inner centromere domain of aligned metaphase II chromosomes after the redistribution of SGOL2, MCAK and INCENP. In this regard, it has been reported that SGOL2, MCAK and INCENP appear as a connecting strand at the inner centromere domain of male mouse prometaphase II chromosomes, but redistribute below sister kinetochores at metaphase II centromeres concomitantly with changes in tension across them (Parra et al. 2003; Parra et al. 2006; Gómez et al. 2007). Consequently, TOCOSs resist changes in tension across the centromeres during chromosome congression at the metaphase II plate.

Strikingly, we have observed that TOCOSs stretch once chromatids have begun their segregation to opposite poles at early anaphase II. The analysis of the relative distribution of Topo II $\alpha$ and the cohesin REC8 during meiosis II showed that both proteins colocalised only at the central region of TOCOSs at the inner centromere domain of metaphase II chromosomes. Then, REC8 disappeared from the inner domain during the metaphase II/anaphase II transition, whereas TOCOSs stretched between the early anaphase II chromatids. These results support that the cleavage of the centromeric cohesin complexes by separase triggers the initial separation of the chromatids, that still remain associated by TOCOSs (Fig. 9). Accordingly, TOCOSs could represent the 
last resistance force counteracting the spindle pulling forces acting on both sister kinetochores after the release of of cohesin, Topo II $\alpha$ cannot resist spindle pulling forces during mitosis (Oliveira et al. 2010) and female mouse meiosis (Tachibana-Konwalski et al. 2010). The subsequent disappearance of TOCOSs during anaphase II suggests that Topo II $\alpha$ finally decatenates the persisting centromeric connections between segregating chromatids. This last assumption is supported by our cytological observation of stretched TOCOSs between the kinetochores of lagging segregating chromatids during anaphase II after the ET-mediated inhibition of the enzymatic activity of Topo II $\alpha$. In this context, we assume that Topo II $\alpha$ has a catalytic activity at the centromeres during the metaphase II/anaphase II transition that promotes the decatenation of hairpin structures formed by satellite centromeric DNA, as previously demonstrated during mitosis (Floridia et al. 2000; Spence et al. 2002; Jonstrup et al. 2008). Altogether, these data indicate that the cleavage of centromeric cohesin complexes is followed by the subsequent decatenating activity of Topo II $\alpha$ during early anaphase II. A similar conclusion has been raised in vertebrate mitotic cells. In this sense, the depletion of the cohesin subunit RAD21 or the shugoshin SGOL1 promotes a premature loss of cohesion, but the simultaneous chemical inhibition or depletion of Topo II $\alpha$ inhibits the release of cohesion (Vagnarelli et al. 2004; Toyoda and Yanagida 2006; Wang et al. 2010). Accordingly, it has been proposed that during mammalian mitosis the centromere DNA decatenation by Topo II $\alpha$ depends on the previous removal of cohesin complexes (Wang et al. 2010). A recent study developed on budding yeast circular minichromosomes also reveals that the presence of cohesin complexes retards Topo II-driven decatenation. Therefore, it is claimed that cohesin complexes regulate cohesion "directly" by embracing sister DNAs inside its rings, and "indirectly" by maintaining catenations that would be only resolved by Topo II once cohesin complexes are removed from chromosomes by a separasemediated mechanism (Farcas et al. 2011).

It has been reported that the helicase PICH appears at centromeric ultrafine anaphase bridges thought to contain unresolved DNA catenations in all cultured cells tested (Baumann et al. 2007; Spence et al. 2007; Wang et al. 2008; Ke et al. 2011; Kaulich et al. 2012). We tested the presence of PICH at the TOCOSs found during early anaphase II, but we did not detect its presence on squashed mouse spermatocytes. Conversely, we detected PICH at ultrafine anaphase bridges in Pam212 mouse somatic cells, but not the presence of Topo II $\alpha$ at such bridges, but only at the centromeres. These results correlate with previous data obtained in HeLa cells where PICH anaphase bridges did not colocalize with Topo II $\alpha$ (Baumann et al. 2007). We therefore suggest that the decatenation activity of Topo II $\alpha$ is differentially regulated during mitotic anaphase and meiotic anaphase II. In this context it is worth noting that since in metaphase II chromosomes the chromatid arms are separated, since arm cohesion was lost by anaphase I, the centromere is the only region that resists the pulling forces acting on sister kinetochores. By contrast, in mitotic metaphase chromosomes sister chromatids remain tightly associated not only at centromeres but also along their arms. Consequently, the pulling forces on sister kinetochores are counteracted by centromere and arm cohesion in metaphase mitotic chromosomes, but only by centromere cohesion in metaphase II chromosomes. These differences could explain two distinct ways of regulation of Topo II $\alpha$-based decatenation at mitotic and meiosis II centromeres.

In summary, taking into account previous and present results on the behaviour of the shugoshin SGOL2, the cohesin REC8 and Topo II $\alpha$, we propose a two-step model for the sequential release of centromeric cohesion during male mammalian meiosis II (Fig. 9). According to this model, the capture of microtubules emanating 
from opposite poles during prometaphase II creates tension across the centromeres, or at both sister kinetochores, that would promote the redistribution of the cohesin protector protein SGOL2 (Gómez et al. 2007; Lee et al. 2008). After the tension-dependent redistribution of SGOL2, the centromeric cohesin complexes would be accessible to the cleavage action of separase. This cleavage would trigger the entry into anaphase II and the chromatids would initiate their segregation to opposite poles. Finally, the removal of cohesin complexes would allow Topo II $\alpha$ to decatenate the persisting centromeric catenations. Further studies need to be performed to clarify the regulation of the decatenating activity of Topo II $\alpha$ during meiosis II, through its phosphorylation and SUMOylation, and the participation of helicases. 


\section{Acknowledgements}

We thank Linda Wordeman, Bill Earnshaw, Jibak Lee and Erich A. Nigg for providing MCAK, INCENP, REC8 and PICH antibodies, respectively; Lorena Barreras for technical assistance; and Kim Nasmyth for providing REC8-myc transgenic male mice. This work was supported by grants BFU2009-10987/BCM to A.V., BFU200908975 to J.L.B., SAF2011-25252 to A.M.P., and SAF2011-28842-C02-01 to J.A.S. from Ministerio de Ciencia e Innovación and Ministerio de Economía y Competitividad (Spain), and grant FMM AP98712012 from Fundación Mutua Madrileña to J.L.B. 


\section{References}

Adelman CA, Petrini JH (2008) ZIP4H (TEX11) deficiency in the mouse impairs meiotic double strand break repair and the regulation of crossing over. PLoS Genet 4:e1000042

Andrews PD, Ovechkina Y, Morrice N, Wagenbach M, Duncan K, Wordeman L, Swedlow JR (2004) Aurora B regulates MCAK at the mitotic centromere. Dev Cell 6:253-268

Baumann C, Korner R, Hofmann K, Nigg EA (2007) PICH, a centromere-associated SNF2 family ATPase, is regulated by Plk1 and required for the spindle checkpoint. Cell 128:101-114

Chan KL, North PS, Hickson ID (2007) BLM is required for faithful chromosome segregation and its localization defines a class of ultrafine anaphase bridges. EMBO J 26:3397-3409

Cobb J, Miyaike M, Kikuchi A, Handel MA (1999) Meiotic events at the centromeric heterochromatin: histone H3 phosphorylation, topoisomerase II $\alpha$ localization and chromosome condensation. Chromosoma 108:412425

Eckley DM, Ainsztein AM, Mackay AM, Goldberg IG, Earnshaw WC (1997) Chromosomal proteins and cytokinesis: patterns of cleavage furrow formation and inner centromere protein positioning in mitotic heterokaryons and midanaphase cells. J Cell Biol 136:1169-1183

Farcas AM, Uluocak P, Helmhart W, Nasmyth K (2011) Cohesin's concatenation of sister DNAs maintains their intertwining. Mol Cell 44:97-107

Floridia G, Zatterale A, Zuffardi O, Tyler-Smith C (2000) Mapping of a human centromere onto the DNA by topoisomerase II cleavage. EMBO Rep 1:489-493

Gómez R, Valdeolmillos A, Parra MT, Viera A, Carreiro C, Roncal F, Rufas JS, Barbero JL, Suja JA (2007) Mammalian SGO2 appears at the inner centromere domain and redistributes depending on tension across centromeres during meiosis II and mitosis. EMBO Rep 8:173-180

Gutiérrez-Caballero C, Herrán Y, Sánchez-Martín M, Suja JA, Barbero JL, Llano E, Pendás AM (2011) Identification and molecular characterization of the mammalian $\alpha$-kleisin RAD21L. Cell Cycle 10:14771487

Gutiérrez-Caballero C, Cebollero LR, Pendás AM (2012) Shugoshins: from protectors of cohesion to versatile adaptors at the centromere. Trends Genet 28:351-360

Hauf S, Roitinger E, Koch B, Dittrich CM, Mechtler K, Peters JM (2005) Dissociation of cohesin from chromosome arms and loss of arm cohesion during early mitosis depends on phosphorylation of SA2. PLoS Biol 3:e69

Herrán Y, Gutiérrez-Caballero C, Sánchez-Martín M, Hernández T, Viera A, Barbero JL, de Álava E, de Rooij DG, Suja JA, Llano E et al (2011) The cohesin subunit RAD21L functions in meiotic synapsis and exhibits sexual dimorphism in fertility. EMBO J 30:3091-3105

Hirano T (2012) Condensins: universal organizers of chromosomes with diverse functions. Genes Dev 26:16591678

Ishiguro K, Kim J, Fujiyama-Nakamura S, Kato S, Watanabe Y (2011) A new meiosis-specific cohesin complex implicated in the cohesin code for homologous pairing. EMBO Rep 12:267-275

Jonstrup AT, Thomsen T, Wang Y, Knudsen BR, Koch J, Andersen AH (2008) Hairpin structures formed by alpha satellite DNA of human centromeres are cleaved by human topoisomerase II $\alpha$. Nucleic Acids Res 36:6165-6174

Kallio M, Lähdetie J (1996) Fragmentation of centromeric DNA and prevention of homologous chromosome separation in male mouse meiosis in vivo by the topoisomerase II inhibitor etoposide. Mutagenesis 11:435443

Kaulich M, Cubizolles F, Nigg EA (2012) On the regulation, function, and localization of the DNA-dependent ATPase PICH. Chromosoma 121:395-408

Ke Y, Huh JW, Warrington R, Li B, Wu N, Leng M, Zhang J, Ball HL, Li B, Yu H (2011) PICH and BLM limit histone association with anaphase centromeric DNA threads and promote their resolution. EMBO J 30:3309-3321

Kitajima TS, Sakuno T, Ishiguro K, Iemura S, Natsume T, Kawashima SA, Watanabe Y (2006) Shugoshin collaborates with protein phosphatase $2 A$ to protect cohesin. Nature 441:46-52

Klein F, Laroche T, Cardenas ME, Hofmann JF, Schweizer D, Gasser SM (1992) Localization of RAP1 and topoisomerase II in nuclei and meiotic chromosomes of yeast. J Cell Biol 117:935-948

Kudo NR, Wassmann K, Anger M, Schuh M, Wirth KG, Xu H, Helmhart W, Kudo H, McKay M, Maro B et al (2006) Resolution of chiasmata in oocytes requires separase-mediated proteolysis. Cell 126:135-146

Lee J, Hirano T (2011) RAD21L, a novel cohesin subunit implicated in linking homologous chromosomes in mammalian meiosis. J Cell Biol 192:263-276

Lee J, Iwai T, Yokota T, Yamashita M (2003) Temporally and spatially selective loss of Rec8 protein from meiotic chromosomes during mammalian meiosis. J Cell Sci 116:2781-2790 
Lee J, Kitajima TS, Tanno Y, Yoshida K, Morita T, Miyano T, Miyake M, Watanabe Y (2008) Unified mode of centromeric protection by shugoshin in mammalian oocytes and somatic cells. Nat Cell Biol 10:42-52

Lee MT, Bachant J (2009) SUMO modification of DNA topoisomerase II: trying to get a CENse of it all. DNA Repair 8:557-568

Llano E, Gómez R, Gutiérrez-Caballero C, Herrán Y, Sánchez-Martín M, Vázquez-Quinones L, Hernández T, de Álava E, Cuadrado A, Barbero JL et al (2008) Shugoshin-2 is essential for the completion of meiosis but not for mitotic cell division in mice. Genes Dev 22:2400-2413

Matulis S, Handel MA (2006) Spermatocyte responses in vitro to induced DNA damage. Mol Reprod Dev 73:1061-1072

Marchetti F, Bishop JB, Lowe X, Generoso WM, Hozier J, Wyrobek AJ (2001) Etoposide induces heritable chromosomal aberrations and aneuploidy during male meiosis in the mouse. Proc Natl Acad Sci USA 98:3952-3957

McNicoll F, Stevense M, Jessberger R (2013) Cohesin in gametogenesis. Curr Top Dev Biol 102:1-34

Murray AW, Szostak JW (1985) Chromosome segregation in mitosis and meiosis. Annu Rev Cell Biol 1:289315

Nasmyth K (2011) Cohesin: a catenase with separate entry and exit gates? Nat Cell Biol 13:1170-1177

Nitiss JL (2009a) DNA topoisomerase II and its growing repertoire of biological functions. Nat Rev Cancer 9:327-337

Nitiss JL (2009b) Targeting DNA topoisomerase II in cancer chemotherapy. Nat Rev Cancer 9:338-350

Oliveira RA, Hamilton RS, Pauli A, Davis I, Nasmyth K (2010) Cohesin cleavage and Cdk inhibition trigger formation of daughter nuclei. Nat Cell Biol 12:185-192

Page J, Suja JA, Santos JL, Rufas JS (1998) Squash procedure for protein immunolocalization in meiotic cells. Chromosome Res 6:639-642

Parra MT, Page J, Yen TJ, He D, Valdeolmillos A, Rufas JS, Suja JA (2002) Expression and behaviour of CENP-E at kinetochores during mouse spermatogenesis. Chromosoma 111:53-61

Parra MT, Viera A, Gómez R, Page J, Carmena M, Earnshaw WC, Rufas JS, Suja JA (2003) Dynamic relocalization of the chromosomal passenger complex proteins inner centromere protein (INCENP) and aurora-B kinase during male mouse meiosis. J Cell Sci 116:961-974

Parra MT, Viera A, Gómez R, Page J, Benavente R, Santos JL, Rufas JS, Suja JA (2004) Involvement of the cohesin Rad21 and SCP3 in monopolar attachment of sister kinetochores during mouse meiosis I. J Cell Sci 117:1221-1234

Parra MT, Gómez R, Viera A, Page J, Calvente A, Wordeman L, Rufas JS, Suja JA (2006) A perikinetochoric ring defined by MCAK and Aurora-B as a novel centromere domain. PLoS Genet 2:e84

Peters AH, Plug AW, van Vugt MJ, de Boer P (1997) A drying-down technique for the spreading of mammalian meiocytes from the male and female germline. Chromosome Res 5:66-68

Porter AC, Farr CJ (2004) Topoisomerase II: untangling its contribution at the centromere. Chromosome Res 12:569-583

Prieto I, Suja JA, Pezzi N, Kremer L, Martínez-A C, Rufas JS, Barbero JL (2001) Mammalian STAG3 is a cohesin specific to sister chromatid arms in meisosis I. Nat Cell Biol 3:761-766

Prieto I, Pezzi N, Buesa JM, Kremer L, Barthelemy I, Carreiro C, Roncal F, Martínez A, Gómez L, Fernández R et al (2002) STAG2 and Rad21 mammalian mitotic cohesins are implicated in meiosis. EMBO Rep 3:543550

Riedel CG, Katis VL, Katou Y, Mori S, Itoh T, Helmhart W, Gálová M, Petronczki M, Gregan J, Cetin B et al (2006) Protein phosphatase 2A protects centromeric sister chromatid cohesion during meiosis I. Nature 441:53-61

Russell LB, Hunsicker PR, Kerley M, Pyle A, Saxton AM (2004) Etoposide exposure during male mouse pachytene has complex effects on crossing-over and causes nondisjunction. Mutat Res 565:61-77

Spence JM, Critcher R, Ebersole TA, Valdivia MM, Earnshaw WC, Fukagawa T, Farr CJ (2002) Co-localization of centromere activity, proteins and topoisomerase II within a subdomain of the major human $\mathrm{X}$ alphasatellite array. EMBO J 21:5269-5280

Spence JM, Phua HH, Mills W, Carpenter AJ, Porter AC, Farr CJ (2007) Depletion of topoisomerase II $\alpha$ leads to shortening of the metaphase interkinetochore distance and abnormal persistence of PICH-coated anaphase threads. J Cell Sci 120:3952-3964

Suja JA, Barbero JL (2009) Cohesin complexes and sister chromatid cohesion in mammalian meiosis. Genome Dyn 5:94-116

Tachibana-Konwalski K, Godwin J, van der Weyden L, Champion L, Kudo NR, Adams DJ, Nasmyth K (2010) Rec8-containing cohesin maintains bivalents without turnover during the growing phase of mouse oocytes. Genes Dev 24:2505-2516

Tang Z, Shu H, Qi W, Mahmood NA, Mumby MC, Yu H (2006) PP2A is required for centromeric localization of Sgo1 and proper chromosome segregation. Dev Cell 10:575-585 
Toyoda Y, Yanagida M (2006) Coordinated requirements of human topo II and cohesin for metaphase centromere alignment under Mad2-dependent spindle checkpoint surveillance. Mol Biol Cell 17:2287-2302

Uhlmann F, Lottspeich F, Nasmyth K (1999) Sister-chromatid separation at anaphase onset is promoted by cleavage of the cohesin subunit Scc1. Nature 400:37-42

Vagnarelli P, Morrison C, Dodson H, Sonoda E, Takeda S, Earnshaw WC (2004) Analysis of Scc1-deficient cells defines a key metaphase role of vertebrate cohesin in linking sister kinetochores. EMBO Rep 5:167171

Viera A, Gómez R, Parra MT, Schmiesing JA, Yokomori K, Rufas JS, Suja JA (2007) Condensin I reveals new insights on mouse meiotic chromosome structure and dynamics. PLoS ONE 2:e783

Wang LH, Schwarzbraun T, Speicher MR, Nigg EA (2008) Persistence of DNA threads in human anaphase cells suggests late completion of sister chromatid decatenation. Chromosoma 117:123-135

Wang LH, Mayer B, Stemmann O, Nigg EA (2010) Centromere DNA decatenation depends on cohesin removal and is required for mammalian cell division. J Cell Sci 123:806-813

Wolf KW, Baumgart K, Winking H (1988) Meiotic association and segregation of the achiasmatic giant sex chromosomes in the male field vole (Microtus agrestis). Chromosoma 97:124-133 


\section{Figure legends}

Fig. 1 Topo II $\alpha$ distribution during meiosis I. Double-immunolabellings of Topo II $\alpha$ (green) with either SYCP3 (pseudocoloured in pink, column 1) or kinetochores (ACA; red, columns 3 and 4), and chromatin counterstaining with DAPI (blue; column 4) on squashed spermatocytes. a-d Pachytene spermatocytes. Topo II $\alpha$ appears at chromocentres. The sex body is indicated $(X Y)$. e-h Metaphase I spermatocytes. Topo II $\alpha$ appears at each centromere region as two large signals each one below each sister kinetochore, and also along the chromatid axes. Note that SYCP3 is present at the inner centromere region and along the interchromatid domain. Two large and round cytoplasmic agglomerates of SYCP3 are indicated (arrowheads). Side-views of selected autosomal bivalents are enlarged in $\mathbf{e}$ and $\mathbf{g}$, and a front-view of an autosomal centromere region is enlarged in e. i-l Anaphase I spermatocytes. Topo II $\alpha$ is detected at the centromere regions partially colocalising with SYCP3 (i) and below the sister kinetochores (j-l), and along the chromatid axes (insert in k). m-p Telophase I spermatocytes. Topo II $\alpha$ is detected at centromere regions partially colocalising with SYCP3 bars (enlargement in $\mathbf{m}$ ), and below sister kinetochores (enlargement in $\mathbf{o}$ ). All images are projections of different focal planes throughout the cell volume. Scale bar $5 \mu \mathrm{m}$

Fig. 2 Relative distribution of Topo II $\alpha$, SGOL2, MCAK, RAD21 and REC8 in metaphase I bivalents. Doubleimmunolabellings of Topo II $\alpha$ (green) with either SGOL2 (red in a, b), MCAK (red in c, d), RAD21 (red in e, f), or REC8 (red in $\mathbf{g}, \mathbf{h}$ ), and chromatin counterstaining with DAPI (blue) on squashed metaphase I spermatocytes. Selected autosomal bivalents (arrows in $\mathbf{a}, \mathbf{e}, \mathbf{g}$ ) are enlarged for each double-immunolabelling, together with their respective schematic illustrations. At each centromere Topo II $\alpha$ is detected as two large signals partially colocalising with the T-shaped labelling of SGOL2, MCAK, and RAD21 at the inner centromere domain. REC8 shows a comma-like distribution in between the Topo II $\alpha$ centromere signals. Note the faint and strong labelling of RAD21 and REC8 at the interchromatid domain, respectively; and the faint Topo II $\alpha$ labelling at the chromatid axes (arrows in the enlargements). The centromeres of the sex chromosomes $(X, Y)$ are indicated in a-f. All images are projections of different focal planes throughout the cell volume. Scale $\operatorname{bar} 5 \mu \mathrm{m}$

Fig. 3 Topo II $\alpha$ distribution during meiosis II. Double-immunolabelling of Topo II $\alpha$ (green) and kinetochores (ACA, red), and chromatin counterstaining with DAPI (blue) on squashed secondary spermatocytes. a-f Interkinesis and prophase II spermatocytes. Topo II $\alpha$ is detected at chromocentres and centromeres, respectively. g-i Metaphase II spermatocyte. Topo II $\alpha$ is localised as a round mass below each sister kinetochore, and as a connecting strand (TOCOS) (arrows) that traverses the centromere region. Two side-viewed metaphase II centromeres are enlarged in $\mathbf{h}$. $\mathbf{j}$-l Early anaphase II spermatocyte. Stretched TOCOSs (arrows) appear between the sister kinetochores of segregating chromatids. m-o Telophase II spermatocyte. Topo II $\alpha$ signals still persist at centromeres. All images are projections of different focal planes throughout the cell volume. Scale bar $3 \mu \mathrm{m}$

Fig. 4 Relative distribution of Topo II $\alpha$, SGOL2, MCAK, RAD21 and REC8 at metaphase II and anaphase II centromeres. Double-immunolabellings of Topo II $\alpha$ (green) with either SGOL2 (red in a, b), MCAK (red in c, d), or RAD21 (red in e, f) on metaphase II spermatocytes, or REC8 (REC8-myc, red) on metaphase II (g, h) and anaphase II (i, j) spermatocytes, and chromatin counterstaining with DAPI (blue). Selected metaphase II 
centromeres are enlarged for each double-immunolabelling, together with their respective schematic illustrations. centromere, SGOL2 and MCAK are detected as two spots at each kinetochore region, RAD21 is not detected, and REC8 appears as a single patch on the TOCOS. i,j Anaphase II spermatocyte. Some thin TOCOSs containing chromatin (arrows) are still connecting the kinetochore regions of segregating chromatids. Segregating chromatids in early anaphase II are schematically illustrated. All images are projections of different focal planes throughout the cell volume of squashed spermatocytes. Scale bar $3 \mu \mathrm{m}$

Fig. 5 Quantitative data on the effects of Topo II $\alpha$ inhibition with ET during meiosis I and II. The percentage of the different categories of alive and apoptotic spermatocytes is indicated for metaphase I (a), anaphase I/telophase I (b), metaphase II (c), and anaphase II/telophase II (d) depending on the duration of the ET treatment with $50 \mathrm{mg} / \mathrm{kg}$. See text for explanation

Fig. 6 Effects of Topo II $\alpha$ inhibition with ET during meiosis I. Double-immunolabelling of Topo II $\alpha$ (green) and kinetochores (ACA; red), and chromatin counterstaining with DAPI (blue) on squashed metaphase I (a-d), anaphase I (e-h), and telophase I (i-l) spermatocytes. The sex chromosomes $(X, Y)$ appear as univalents in the metaphase I shown in a-d. Topo II $\alpha$ appears at the centromeres and also as TOCOSs (arrows) between the centromere regions of lagging homologues in anaphase I and telophase I. Note the presence of two sister kinetochores (arrowheads) at each centromere. The TOCOS detected in telophase I shows chromatin (yellow arrows). All images are projections of different focal planes throughout the cell volume. Scale bar $3 \mu \mathrm{m}$

Fig. 7 Effects of Topo II $\alpha$ inhibition with ET during meiosis II. Double-immunolabelling of Topo II $\alpha$ (green) and kinetochores (ACA; red), and chromatin counterstaining with DAPI (blue) on squashed metaphase II (a-d), anaphase II (e-h), and telophase II (i-n) spermatocytes. A single unaligned Y chromatid $(Y)$ is present in the metaphase II shown in a-d. Stretched TOCOSs (arrows) are observed between sister kinetochores (arrowheads) of lagging chromatids in anaphase II and telophase II (e-n). Note that these TOCOSs colocalise with very thin chromatin threads between the body of both lagging chromatids (yellow arrow in $\mathbf{k}$ ) or between the kinetochore and the body of the chromatids (purple arrows in $\mathbf{k}, \mathbf{n}$ ). All images are projections of different focal planes throughout the cell volume. Scale bar $3 \mu \mathrm{m}$

Fig. 8 Topo II $\alpha$ distribution during meiotic divisions of $\mathrm{Sgol}^{-/}$male mice and $\mathrm{Sgol}^{-/}$male mice treated with ET. Double-immunolabelling of Topo II $\alpha$ (green) and kinetochores (ACA; red), and chromatin counterstaining with DAPI (blue) on squashed metaphase I (a-d), anaphase I (e-h), and metaphase II-like (i-l) spermatocytes. Topo II $\alpha$ appears as a large signal below each kinetochore. Side-views of selected autosomal metaphase I, anaphase I and metaphase II-like centromeres are enlarged in $\mathbf{a}, \mathbf{e}, \mathbf{g}, \mathbf{i}, \mathbf{k}$. All images are projections of different focal planes throughout the cell volume. Scale bar $3 \mu \mathrm{m}$

Fig. 9 Model reflecting the distribution of Topo II $\alpha$ in pachynema and metaphase I, and the removal of cohesin complexes and the Topo II $\alpha$ decatenating activity during the second meiotic division. Kinetochore microtubules are indicated in turquoise, kinetochores in red, Topo II $\alpha$ in green, shugoshin SGOL2 in pink, and the cohesin 
subunit REC8 in blue. The colocalisation of REC8 and SGOL2 is shown in light blue. One chromosome of each bivalent is depicted in light grey and its homologue is darker grey. Chromosomes are telocentric, and the metaphase I bivalent shows a single interstitial chiasma. In pachynema Topo II $\alpha$ decorates the chromocentres while REC8 is located along the cohesin axes beneath the LEs of the SC of the autosomal bivalents and the AE of the sexual bivalent XY. Note that to simplify not all the 19 autosomal bivalents are represented. In metaphase I, Topo II $\alpha$ localizes at two rounded accumulations below the closely associated sister kinetochores, and partially colocalizes with the middle region of the T-shaped SGOL2 structure at each centromere. Topo II $\alpha$ is also located along the chromatid axes. REC8-containing cohesin complexes are also depicted as patches at the interchromatid domain. A hypothetical model accounting for the distribution of Topo II $\alpha$ and REC8-containing cohesin complexes in relation to radial chromatin loops in a mouse metaphase I bivalent is shown. The longitudinal and transverse sections of the arms correspond to areas indicated in the metaphase I bivalent represented. During the prometaphase II/metaphase II transition SGOL2 redistributes unmasking centromeric REC8-containing cohesin complexes. During the metaphase II/anaphase II transition the centromeric cohesin complexes are cleaved by separase before the Topo II $\alpha$ decatenating activity at TOCOs occur. 


\section{Supplementary material}

Fig. S1 Distribution of Topo II $\alpha$ and SYCP3 in metaphase I and anaphase I chromosomes. Doubleimmunolabelling of Topo II $\alpha$ (green) and SYCP3 (red) in selected metaphase I bivalents (a, b) and an anaphase I chromosome (c) from spreaded spermatocytes. Topo II $\alpha$ appears enriched at centromeres and also along the chromatid axes (arrows), while SYCP3 is accumulated at the inner centromere domain and also appears at the interchromatid domain although the labelling interrupts at the chiasma sites (arrowheads). Scale bar $2.5 \mu \mathrm{m}$

Fig. S2 Relative distribution of Topo II $\alpha$ and INCENP during chromosome congression to the metaphase II plate. Double-immunolabelling of Topo II $\alpha$ (green) and INCENP (red) on squashed prometaphase II (a, b) and metaphase II (c, d) spermatocytes. INCENP redistributes from a band traversing the inner centromere domain (a, b) to a single signal at the kinetochore region $(\mathbf{c}, \mathbf{d})$, while Topo II $\alpha$ persists as a TOCOS, as indicated in the schematic illustrations. Scale bar $0.5 \mu \mathrm{m}$

Fig. S3 Distribution of PICH and Topo II $\alpha$ in early anaphase. Double-immunolabelling of PICH (green) and Topo II $\alpha(r e d)$, and chromatin counterstaining with DAPI (blue) on Pam212 early anaphases. Elongated PICH strands are observed between Topo II $\alpha$ signals at the centromeres. Scale bar $3 \mu \mathrm{m}$

Fig. S4 Topo II $\alpha$ distribution during spermatogonial mitosis. a-h Double-immunolabelling of Topo II $\alpha$ (green) and kinetochores (ACA; red), and chromatin counterstaining with DAPI (blue) in spermatogonial prophase and metaphase from squashed seminiferous tubules. i-l Immunolabelling of Topo II (green) and chromatin counterstaining with DAPI (blue) on a spreaded spermatogonial metaphase. Topo II $\alpha$ appears enriched at the centromere regions and faintly represented at the chromatid axes. Two enlarged chromosomes are shown together with a schematic illustration (k,l). Scale bars $3 \mu \mathrm{m}$ in $\mathbf{h}, 5 \mu \mathrm{m}$ in $\mathbf{j}$

Fig. S5 Apoptosis after Topo II $\alpha$ inhibition with ET during meiosis I and meiosis II. Double-immunolabelling of Topo II $\alpha$ (green) and kinetochores (ACA; red); and chromatin counterstaining with DAPI (blue) on squashed spermatocytes. Positive immunoreactivity can only be detected in alive metaphase I (a) and metaphase II (k) spermatocytes. b-f Apoptotic metaphase I spermatocytes with hypercompacted chromosomes without apparent univalents (b, e) and with univalents (arrowheads in $\mathbf{c}, \mathbf{d}, \mathbf{f})$. g,h Apoptotic anaphase I/telophase I spermatocytes with lagging chromosomes (g) and without lagging chromosomes (h). i,j Apoptotic metaphase I spermatocytes with fragmented chromosomes. l-o Apoptotic metaphase II spermatocytes with aligned chromosomes (l-n) and misaligned chromosomes (o). p-y Apoptotic anaphase II/telophase II spermatocytes without lagging chromosomes (p, r, u, w, x), with lagging chromosomes (arrowheads) and chromatin bridges (arrows) (q, s, t, $\mathbf{v})$, and with fragmented chromosomes (y). Scale bar $3 \mu \mathrm{m}$

Fig. S6 TUNEL assay after Topo II $\alpha$ inhibition with ET. TUNEL assay (green) and chromatin counterstaining with DAPI (blue) on squashed spermatocytes after 24 hours of ET treatment. Apoptotic cells correspond to those with hypercondensed chromosomes. The image is a projection of different focal planes throughout the cell volume. Scale bar $5 \mu \mathrm{m}$ 
Movie 1 Metaphase I spermatocyte shown in Fig. 1f-h. Double-immunolabelling of Topo II $\alpha$ (green) and kinetochores (red), and chromatin counterstaining with DAPI (blue)

Movie 2 Metaphase II spermatocyte shown in Fig. 3g-i. Double-immunolabelling of Topo II $\alpha$ (green) and kinetochores (red), and chromatin counterstaining with DAPI (blue)

Movie 3 Early anaphase II spermatocyte. Immunolabelling of Topo II $\alpha$ (green), and chromatin counterstaining with DAPI (blue)

Movie 4 Anaphase I and telophase I spermatocytes after ET treatment shown in Figures 6e-1. Doubleimmunolabelling of Topo II $\alpha$ (green) and kinetochores (red), and chromatin counterstaining with DAPI (blue)

Movie 5 Anaphase II spermatocyte after ET treatment shown in Fig. 7e-h. Double-immunolabelling of Topo II $\alpha$ (green) and kinetochores (red), and chromatin counterstaining with DAPI (blue)

Movie 6 Telophase II spermatocyte after ET treatment shown in Fig. 7i-k. Double-immunolabelling of Topo II $\alpha$ (green) and kinetochores (red), and chromatin counterstaining with DAPI (blue)

Movie 7 Late telophase II spermatocyte after ET treatment shown in Fig. 71-n. Double-immunolabelling of Topo II $\alpha$ (green) and kinetochores (red), and chromatin counterstaining with DAPI (blue) 
Click here to download high resolution image

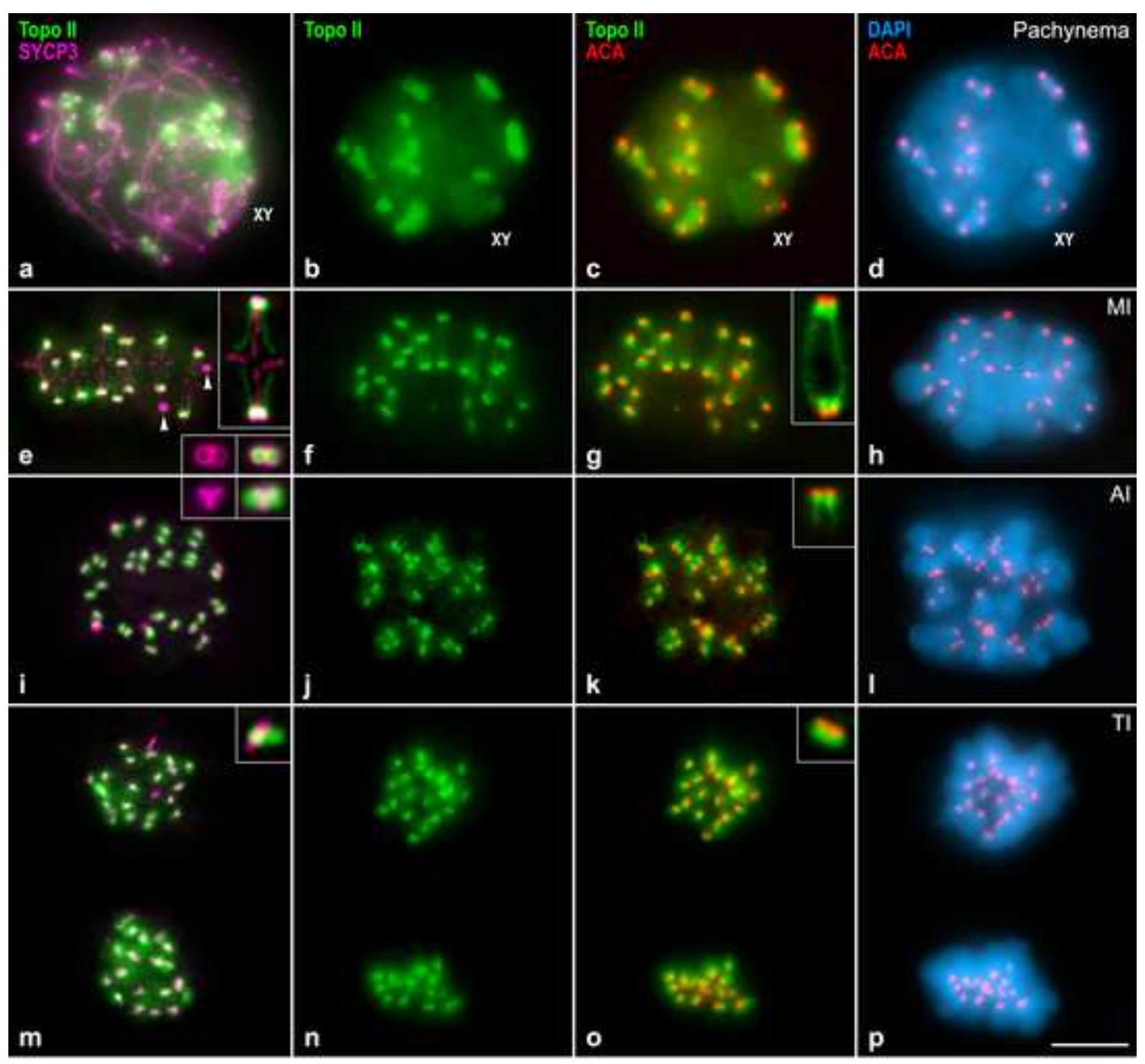

Figure 1 


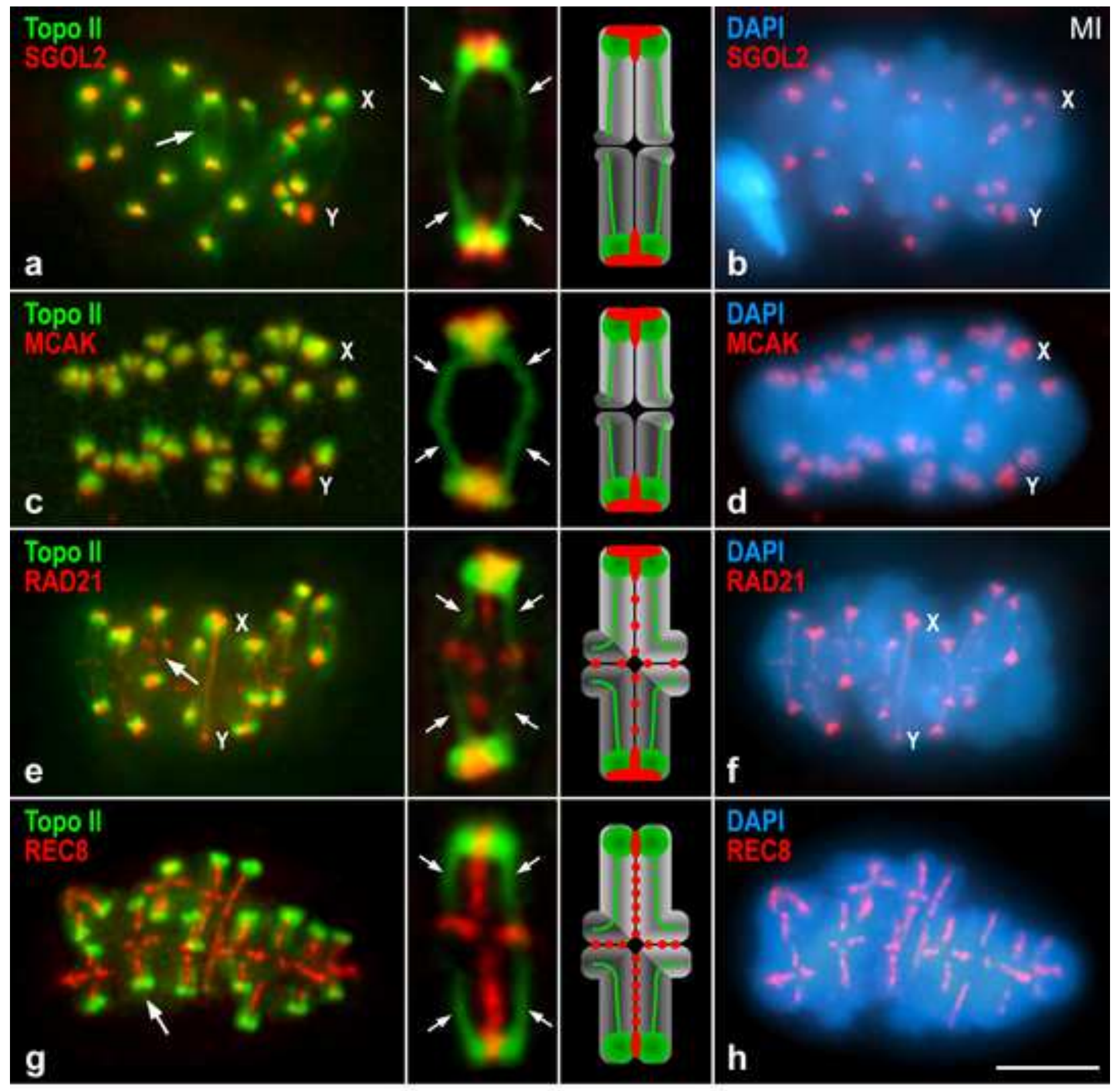

Figure 2 
Click here to download high resolution image

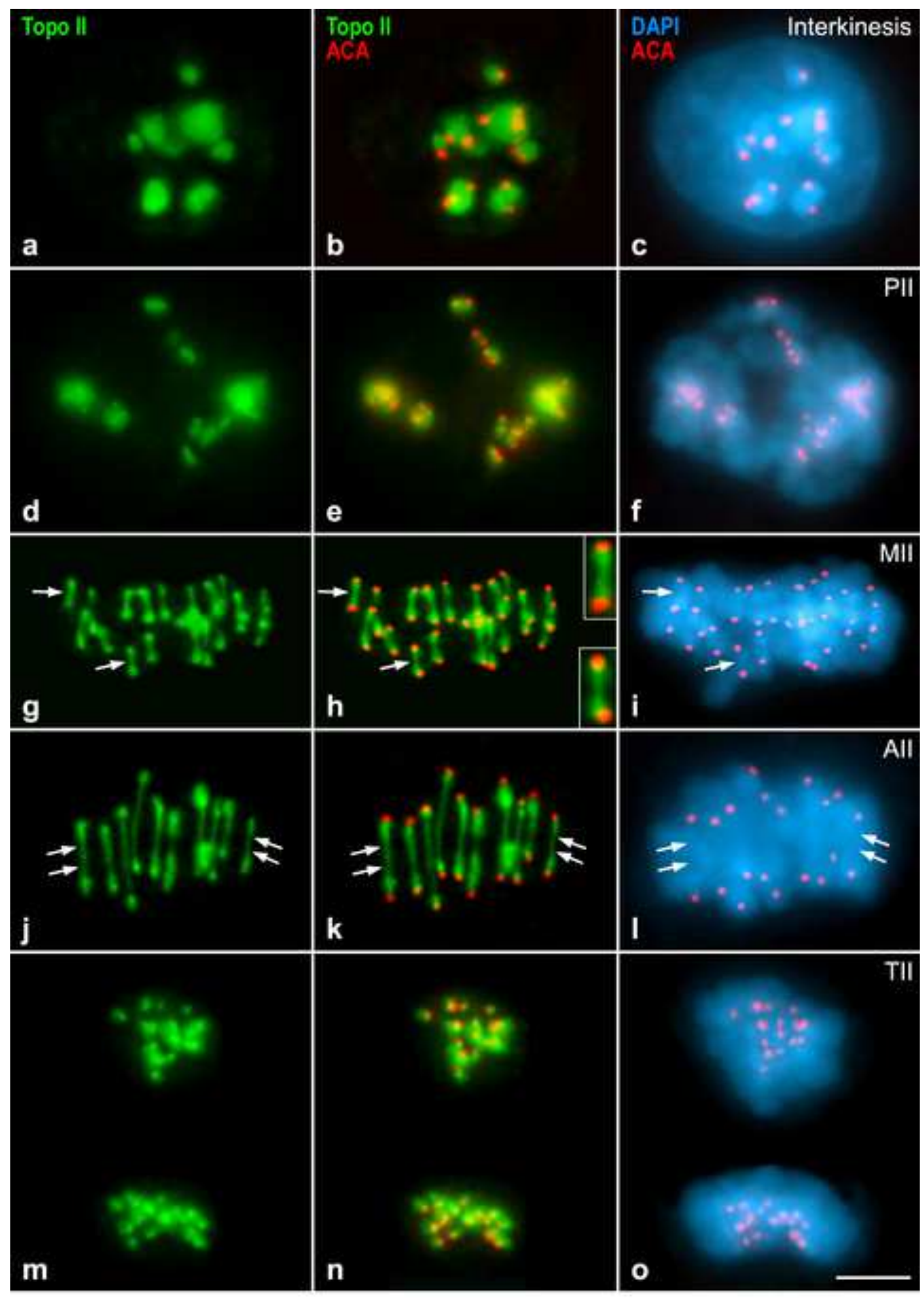

Figure 3 


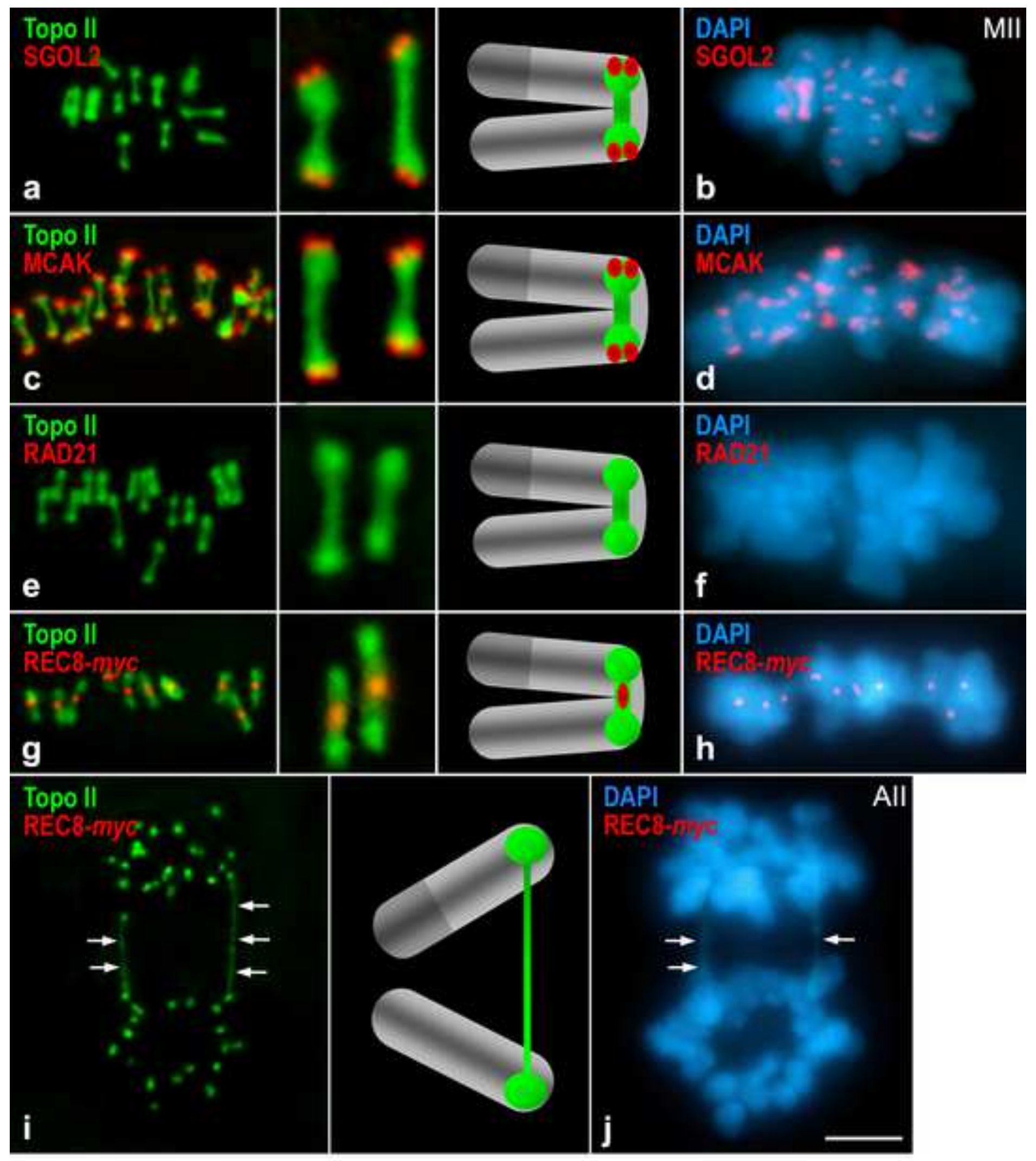

Figure 4 

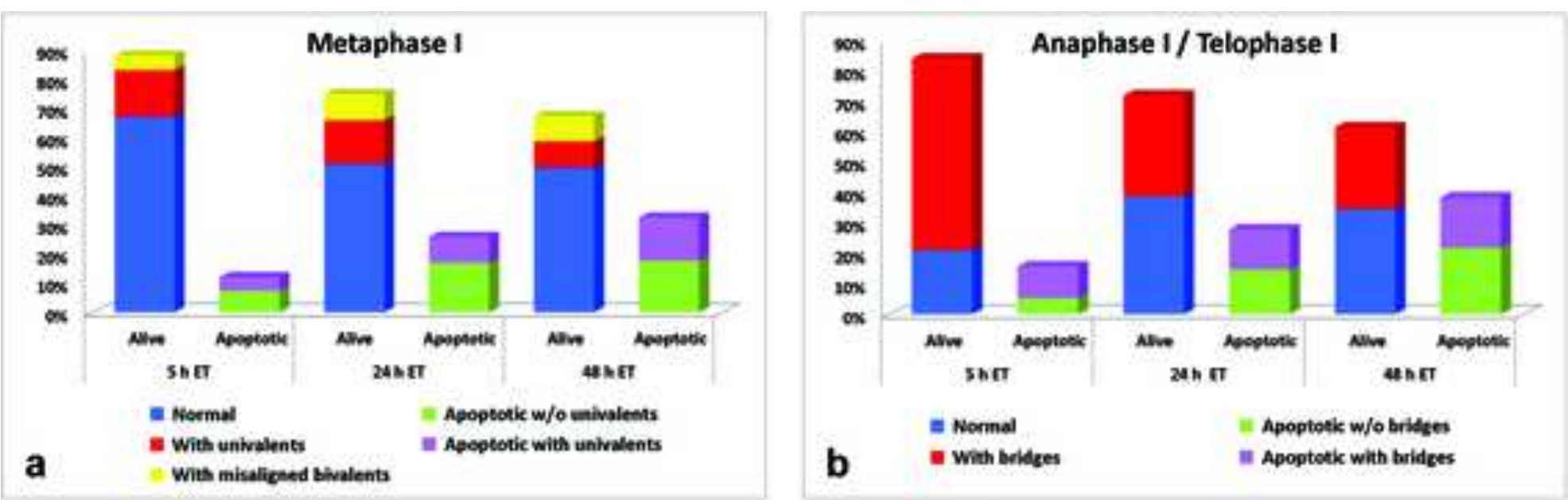

\begin{tabular}{|c|c|c|c|c|c|c|}
\hline Motaphase I & Normal & $\begin{array}{c}\text { Whath } \\
\text { univalents }\end{array}$ & $\begin{array}{l}\text { With } \\
\text { minaliped } \\
\text { blvalionts }\end{array}$ & $\begin{array}{c}\text { Apoptotic }=60 \\
\text { univilents }\end{array}$ & $\begin{array}{l}\text { Apoptotic with } \\
\text { unlvalents }\end{array}$ & $n$ \\
\hline $5 \mathrm{hET}$ & $67,16 \mathrm{~S}$ & $16,05 \%$ & 4.905 & $7,40 \%$ & 4.705 & 406 \\
\hline $24 \mathrm{hET}$ & $50.00 \%$ & $14,50 \%$ & $6.70 \mathrm{~s}$ & $16.80 \%$ & 9.10\% & 309 \\
\hline EShET & $19.68 x$ & $0.05 \%$ & $8 \cos x$ & $17 \operatorname{esc}$ & $1494 x$ & 475 \\
\hline
\end{tabular}

\begin{tabular}{|c|c|c|c|c|c|}
\hline $\begin{array}{l}\text { Anaphase If } \\
\text { Telophase if }\end{array}$ & Nomial & $\begin{array}{l}\text { Weh } \\
\text { bridpet }\end{array}$ & $\begin{array}{l}\text { Apeptotic wilo } \\
\text { bridgess }\end{array}$ & $\begin{array}{c}\text { Apoptotic with } \\
\text { Bridges }\end{array}$ & $n$ \\
\hline SAET & 21,0 & $63.2 \%$ & 5.35 & 10,55 & 10 \\
\hline $24 \mathrm{hET}$ & $3,7 \%$ & 23.35 & $4.7 \%$ & $13 x$ & 75 \\
\hline $48 \mathrm{hET}$ & $\operatorname{sex}$ & 26.95 & 21.85 & 1678 & 78 \\
\hline
\end{tabular}
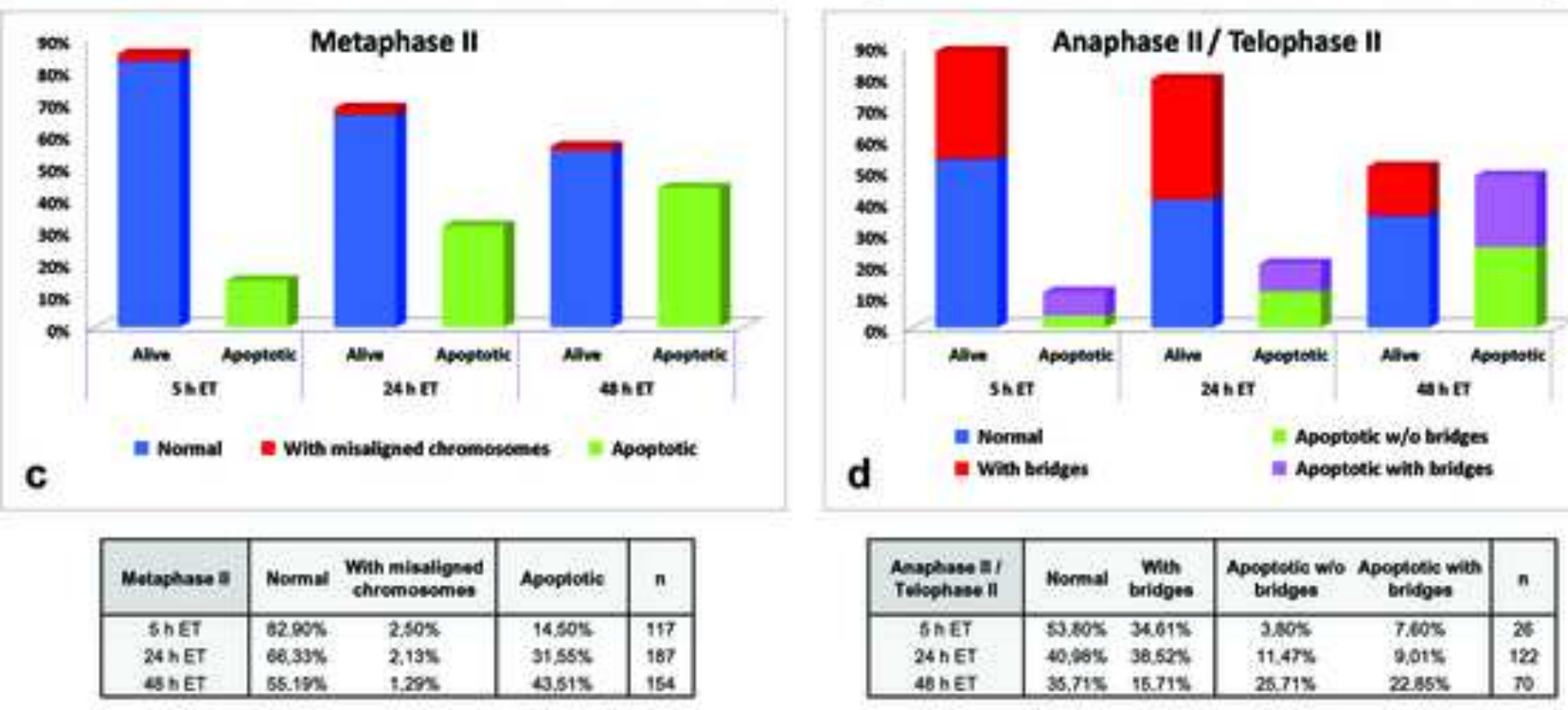

Figure 5 
Topo II inhibition with ET

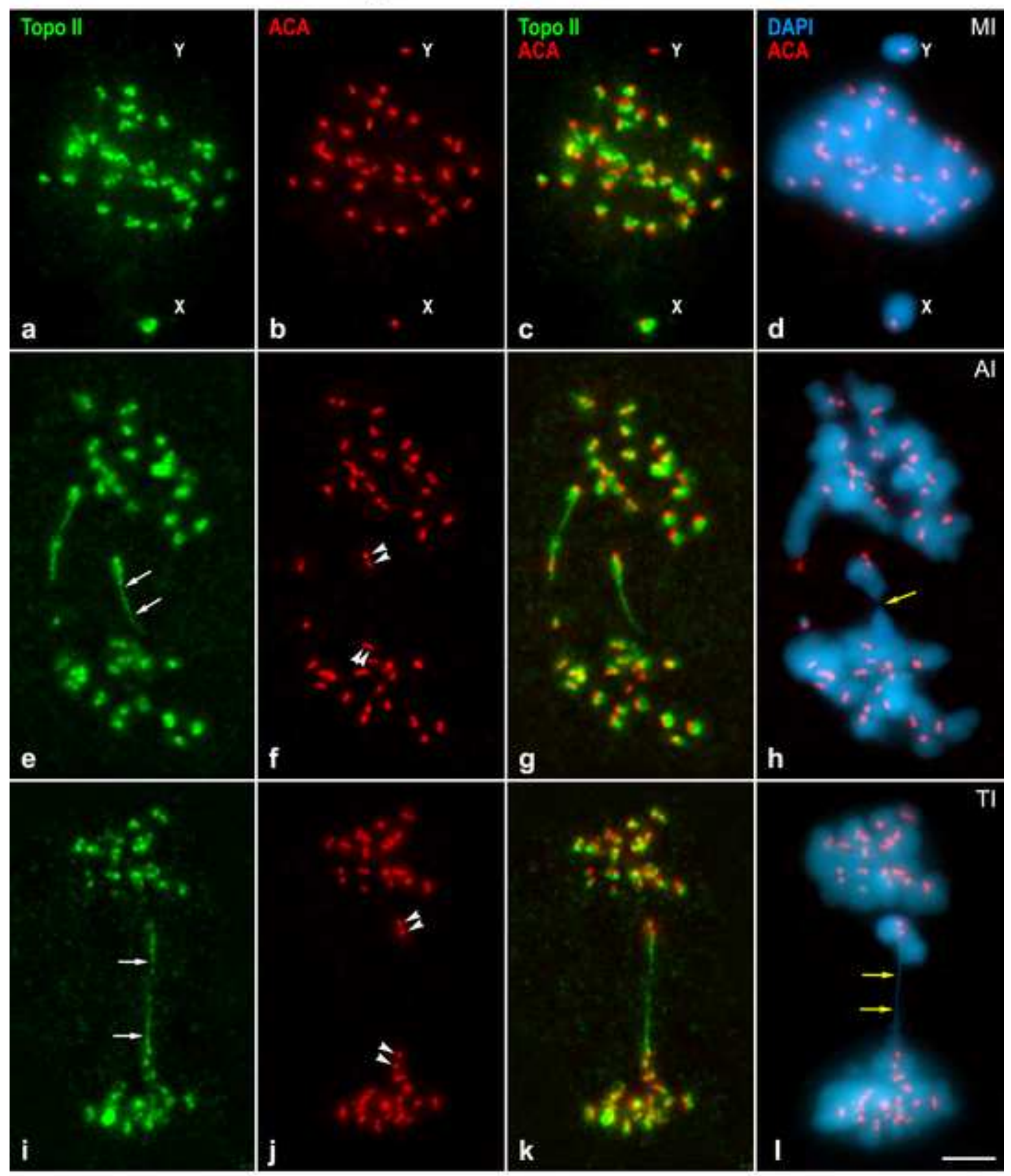

Figure 6 
Topo II inhibition with ET

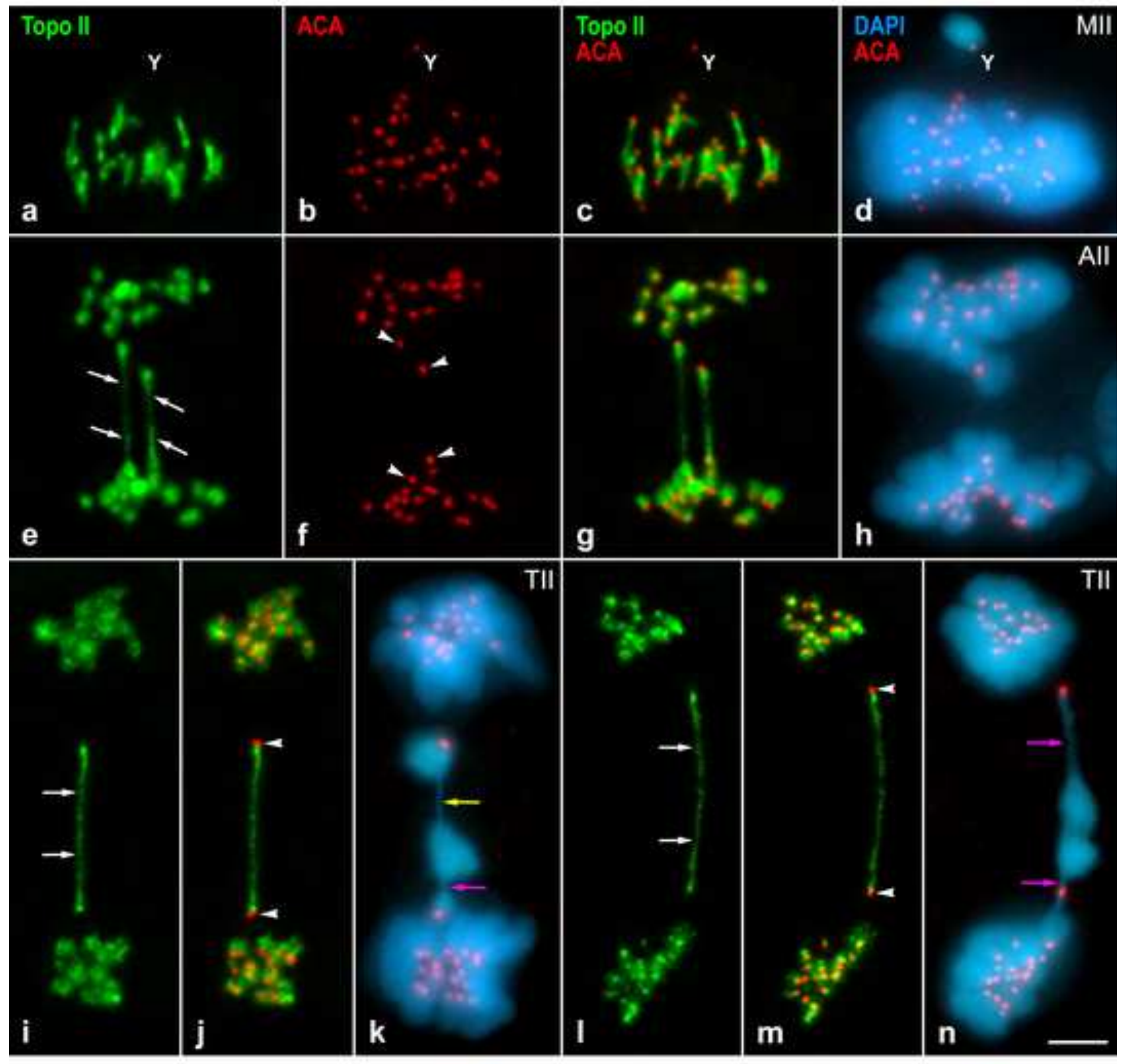

Figure 7 


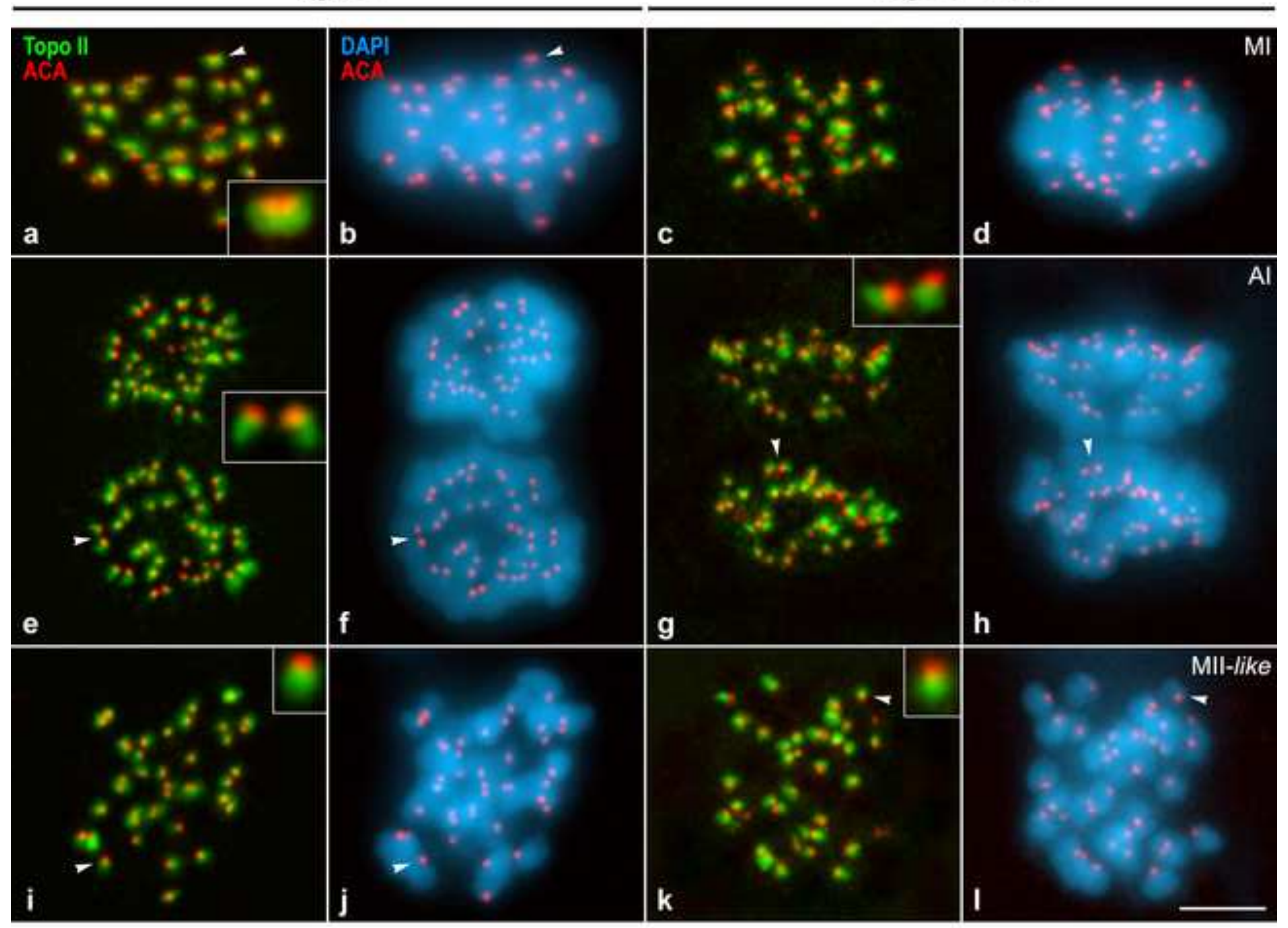

Figure 8 


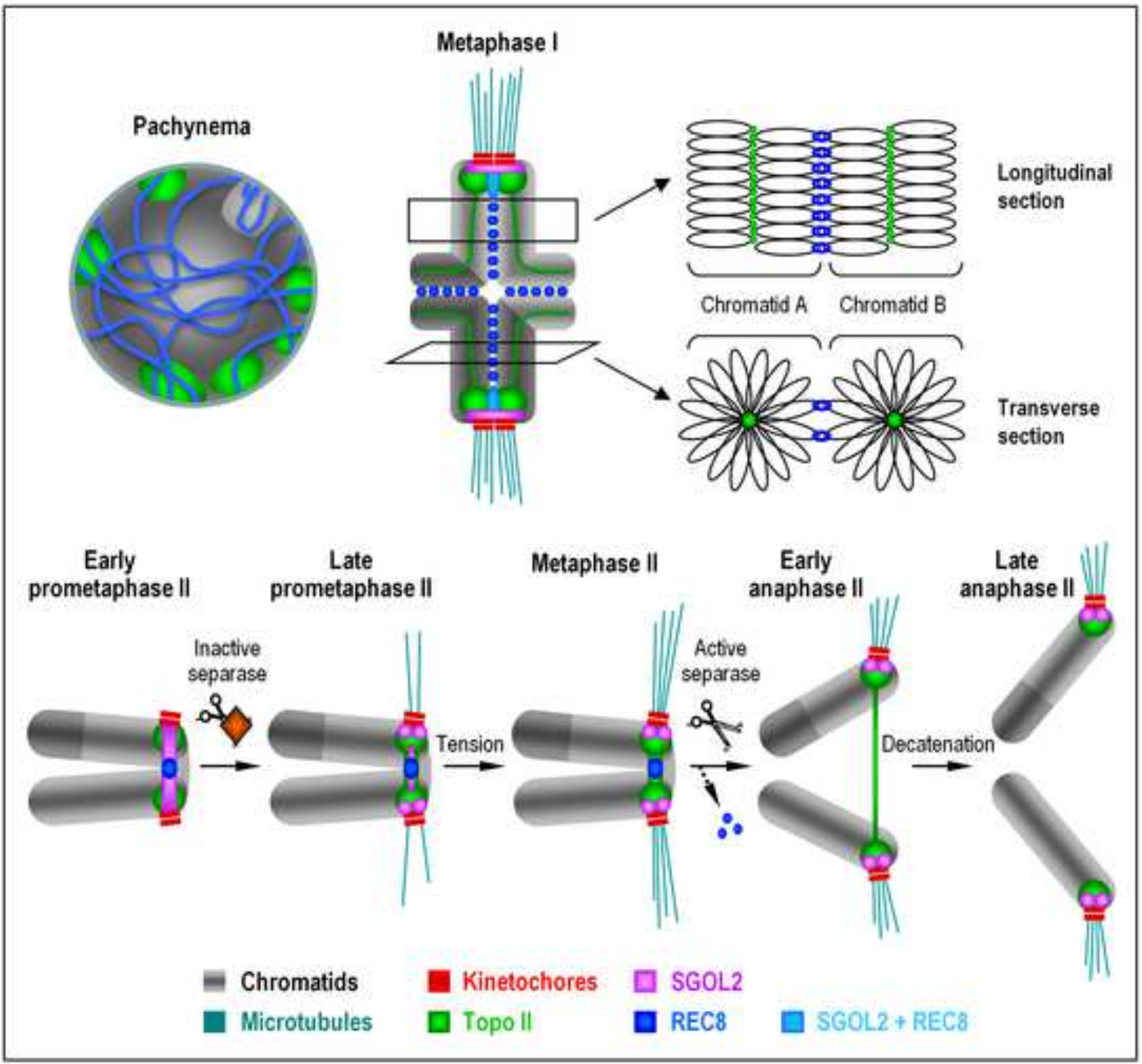

Figure 9 


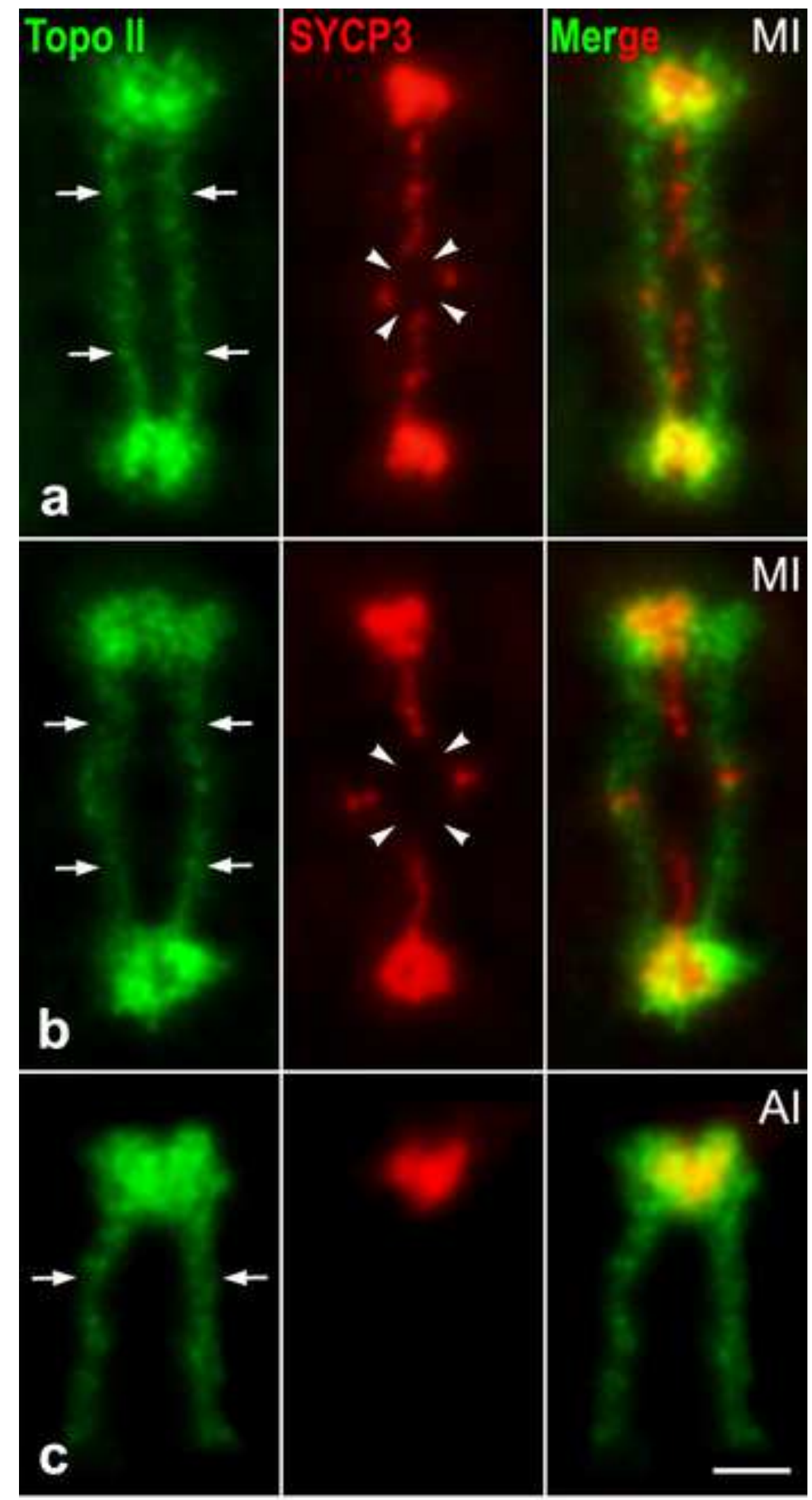

\section{Supplementary Figure 1}




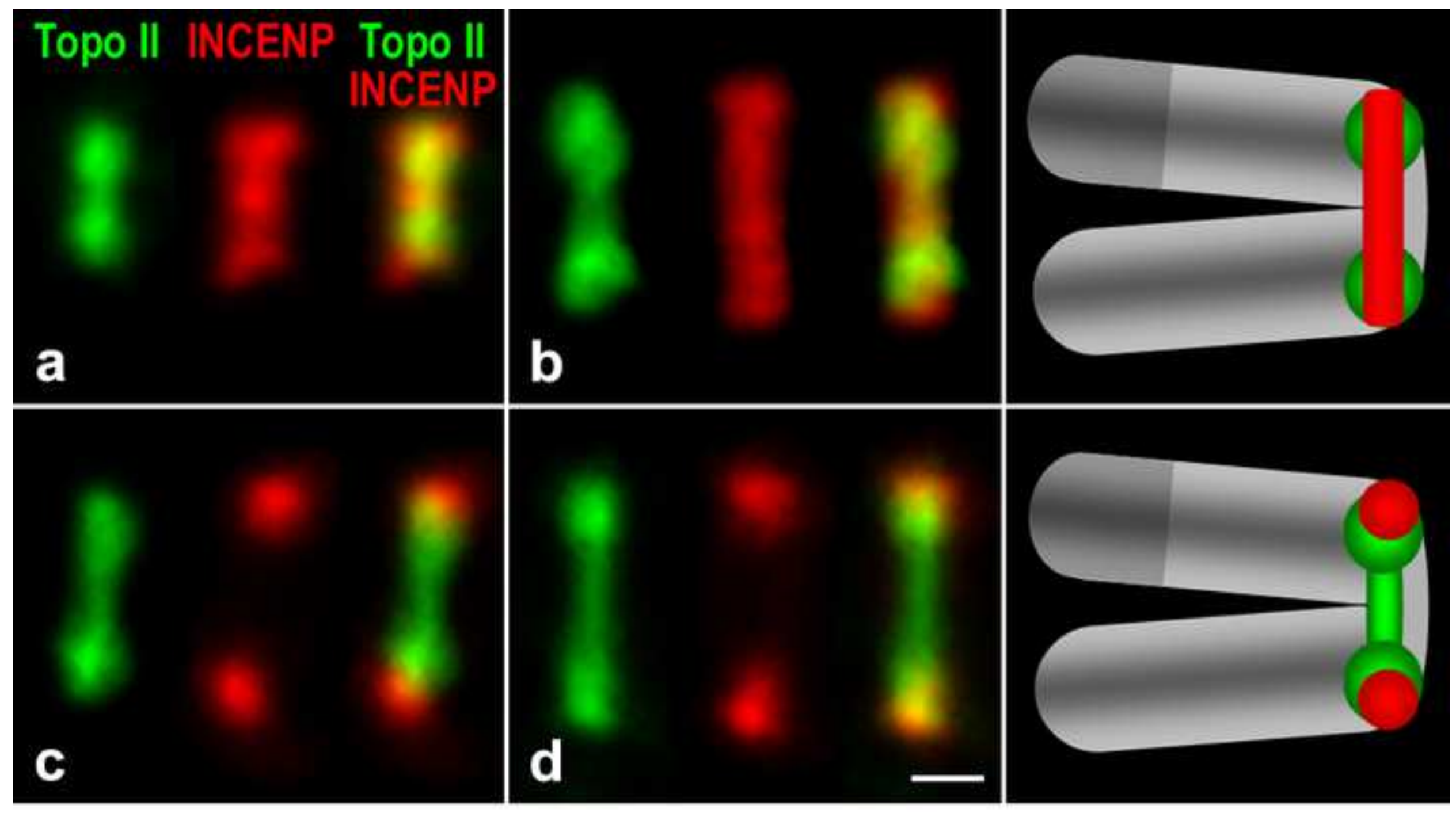

\section{Supplementary Figure 2}


Click here to download high resolution image

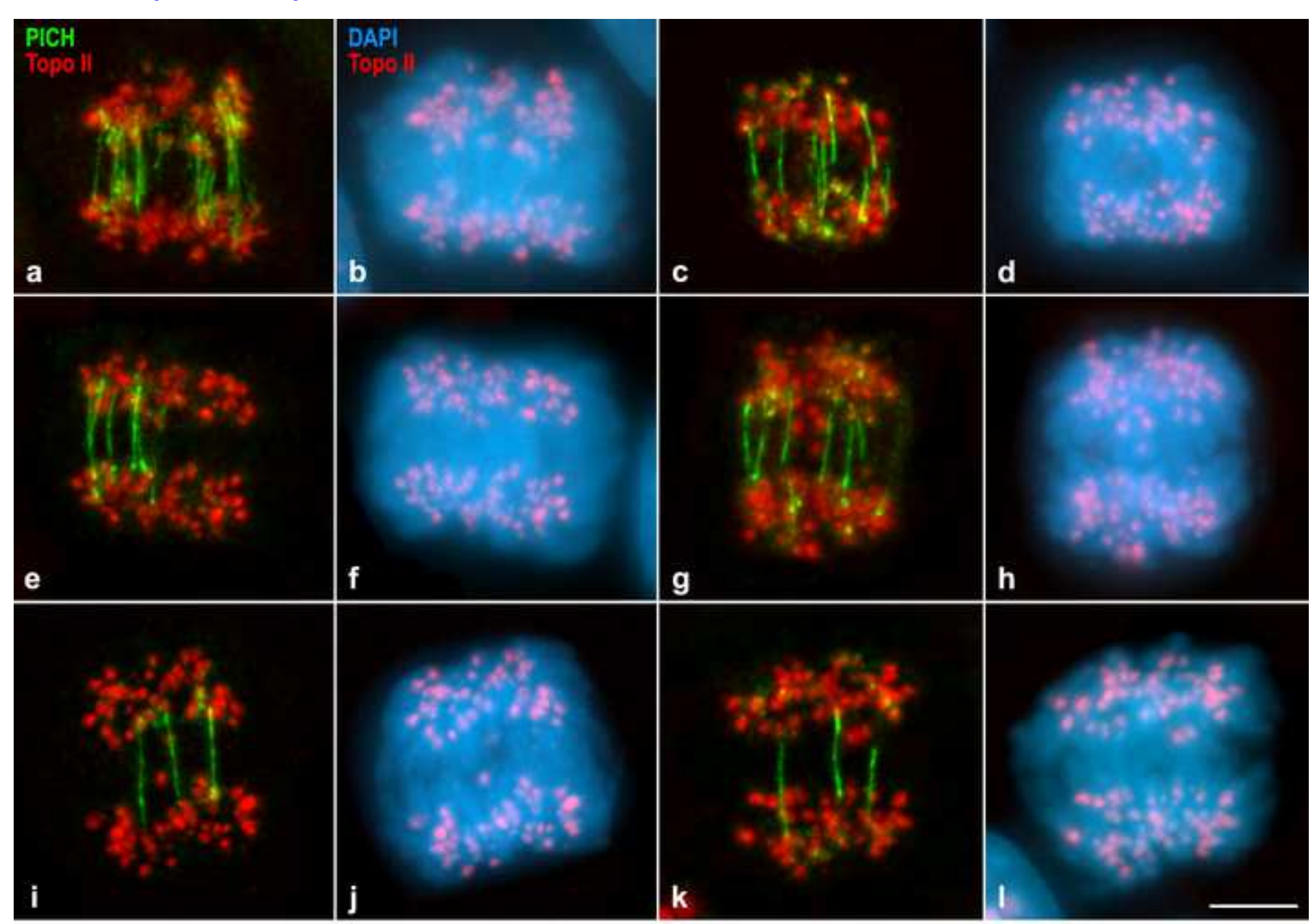

Supplementary Figure 3 


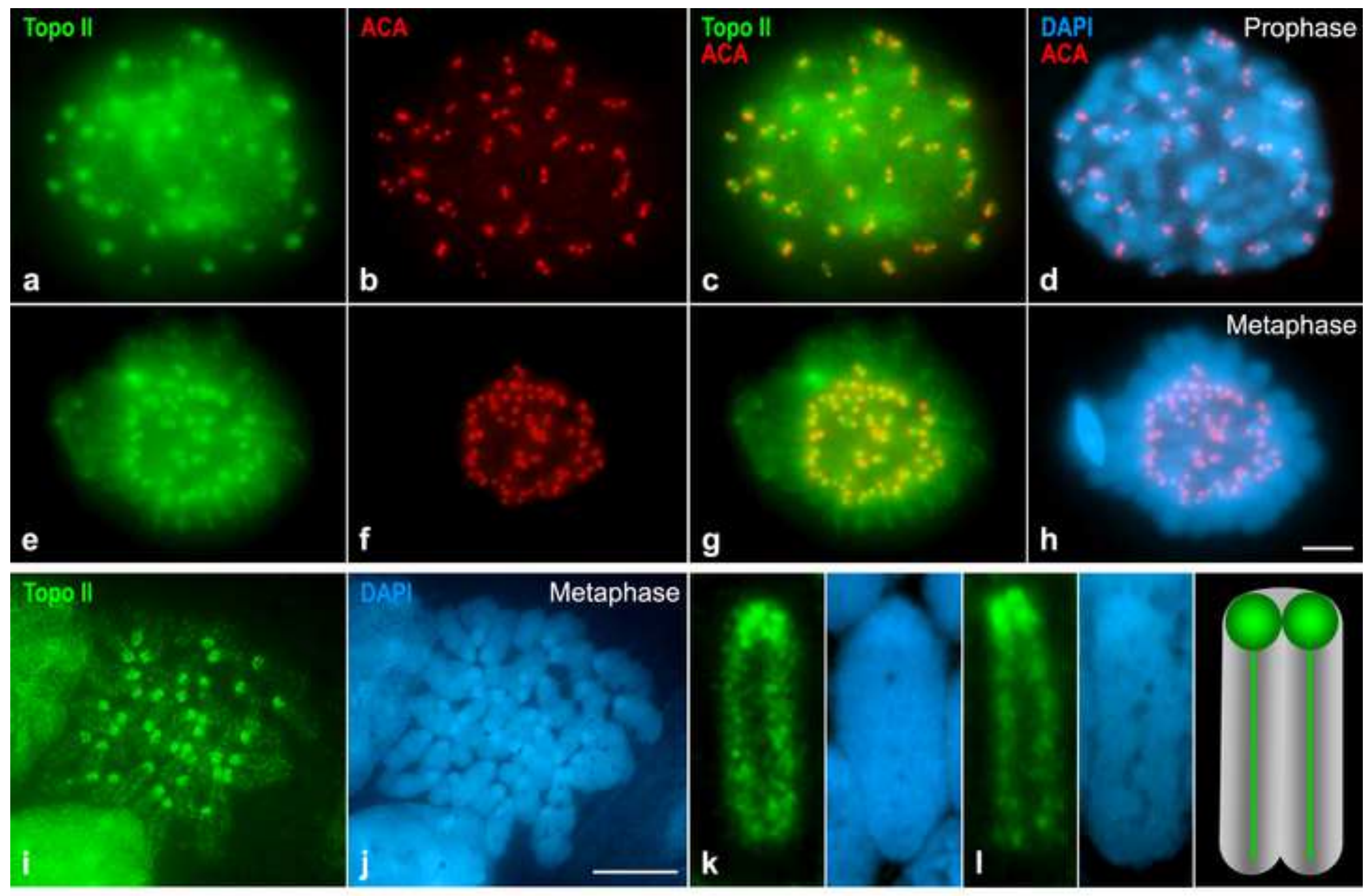

Supplementary Figure 4 


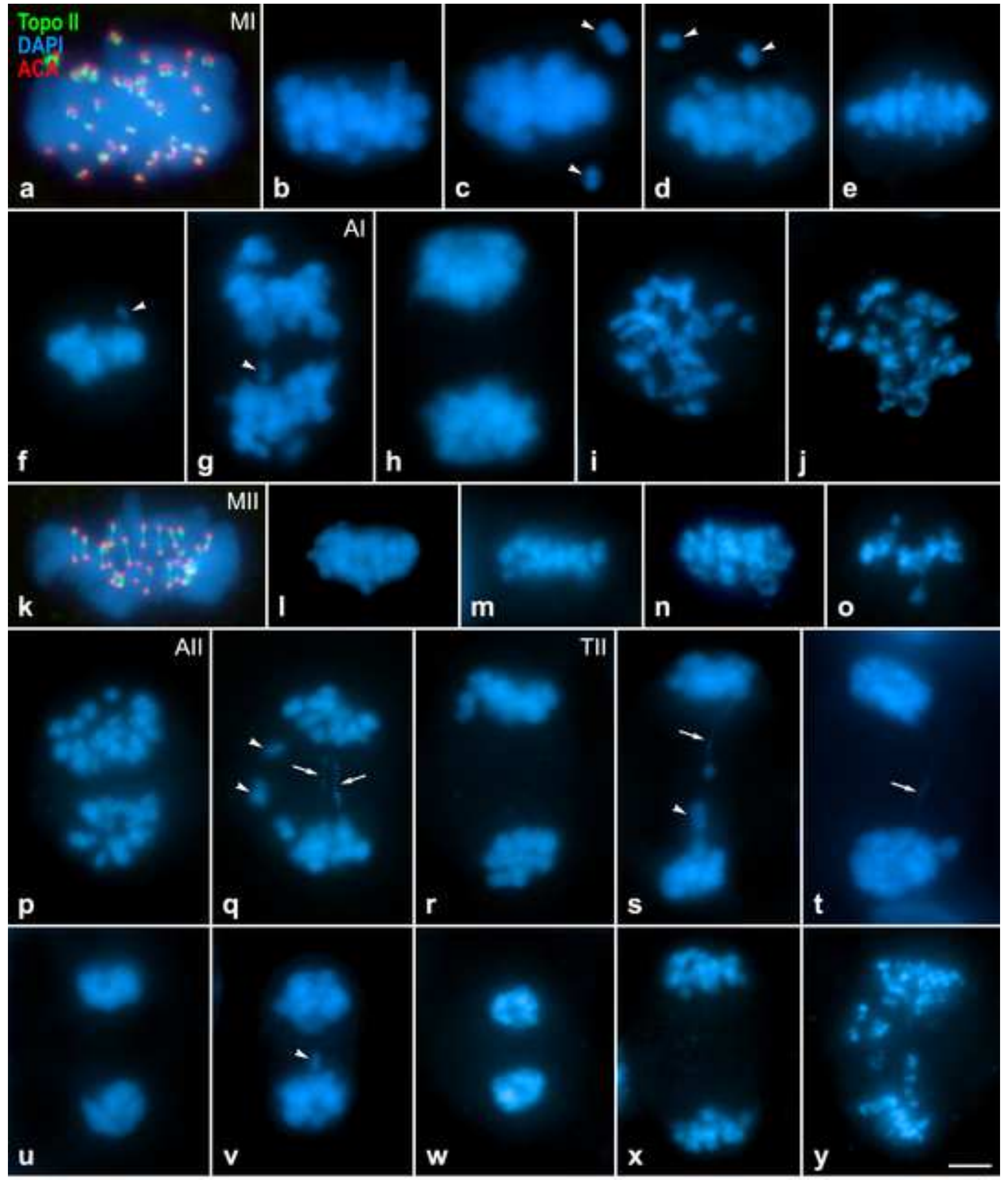

Supplementary Figure 5 


\section{Topo II inhibition with ET}

\section{TUNEL DAPI}

\section{Supplementary Figure 6}

